Geometry $\&$ Topology

Volume 9 (2005) 991-1042

Published: 1 June 2005

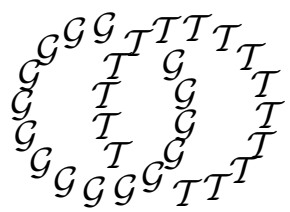

\title{
On the Ozsváth-Szabó invariant of negative definite plumbed 3-manifolds
}

\author{
ANDRÁS NÉMETHI \\ Department of Mathematics \\ Ohio State University \\ Columbus, $\mathrm{OH} 43210$, USA \\ and \\ Rényi Institute of Mathematics \\ Budapest, Hungary \\ Email: nemethi@math.ohio-state.edu, nemethi@renyi.hu \\ URL: http://www.math.ohio-state.edu/ nemethi/
}

\begin{abstract}
The main goal of the present article is the computation of the Heegaard Floer homology introduced by Ozsváth and Szabó for a family of plumbed rational homology 3-spheres. The main motivation is the study of the Seiberg-Witten type invariants of links of normal surface singularities.
\end{abstract}

AMS Classification numbers Primary: 57M27, 57R57

Secondary: 14E15, 14B15, 14J17, 32S25, 32S45

Keywords: 3-manifolds, Ozsváth-Szabó Heegaard Floer homology, SeibergWitten invariants, Seifert manifolds, Lens spaces, Casson-Walker invariant, $\mathbb{Q}-$ homology spheres, Reidemeister-Turaev torsion, normal surface singularities, rational singularities, elliptic singularities

Proposed: Peter Ozsvath

Seconded: Walter Neumann, Tomasz Mrowka
Received: 22 August 2004

Accepted: 13 April 2005 


\section{Introduction}

The main goal of the present article is the computation of the Ozsváth-Szabó $\mathbb{Z}[U]$-module (Heegaard Floer homology) $H F^{+}(M,[k])$ [17, 18, 19, 20] for a family of plumbed rational homology 3 -spheres $M$ and any $\operatorname{spin}^{c}$-structure $[k] \in \operatorname{Spin}^{c}(M)$. The author's main motivation is the study of the SeibergWitten type invariants of links of normal surface singularities.

More precisely, we consider that family of connected, negative definite plumbing graphs - we call them $A R$-graphs (see Section 8) - which satisfy the following property: there exists a vertex such that decreasing the decoration (the Euler number) of that vertex we get a rational graph (in the sense of Artin). This class is surprisingly large: it contains the links of rational and weakly elliptic singularities, the graphs considered by Ozsváth and Szabó in 21, in particular, all the Seifert manifolds (associated with negative definite plumbing graphs). For such plumbing graphs $\Gamma$ (and associated plumbed 3-manifolds $M(\Gamma)$ ) we prove (extending the main result of [21]) the completely topological description $H F^{+}(M(\Gamma),[k])=\mathbb{H}^{+}(\Gamma,[k])$, where $\mathbb{H}^{+}(\Gamma,[k])$ is the combinatorial $\mathbb{Z}[U]-$ module introduced in [21].

Moreover, for such graphs, we provide a precise combinatorial formula (algorithm) for $\mathbb{H}^{+}(\Gamma,[k])$. In order to do this, we define a "graded root" $\left(R_{k}, \chi_{k}\right)$ associated with any connected, negative definite plumbing graph $\Gamma$ and characteristic element $k$. This object connects in a mysterious way two different types of properties, objects and invariants: those coming from the Ozsváth-Szabó (or Seiberg-Witten) theory with those coming from algebraic geometry and singularity theory. For example, its grading $\chi_{k}$ is in fact a Riemann-Roch formula, which guides (modulo a shift) the absolute grading of $\mathrm{HF}^{+}(M,[k])$. We believe that $\left(R_{k}, \chi_{k}\right)$ is the right object which guides the hierarchy of the topological types of links of normal surface singularities (see, for example, Section [6); and at the same time, it contains all the information about $\mathbb{H}^{+}(\Gamma,[k])$. In the body of the paper, for any $A R$ graph, we determine all these "graded roots", we read from them the combinatorial modules $\mathbb{H}^{+}(\Gamma,[k])$, and we compute the numerical invariants $\mathbf{s w}_{M,[k]}^{O S Z}$ which are the candidates provided by the Ozsváth-Szabó theory for the Seiberg-Witten invariants.

We exemplify the theory with a detailed discussion of the lens spaces and Seifert manifolds. Additionally, in these two cases we verify that $\mathbf{s w}_{M,[k]}^{O S z}$ coincides with the Reidemeister-Turaev sign-refined torsion (function) normalized by the Casson-Walker invariant - a fact conjectured by Ozsváth and Szabó.

Examples show that the above results about $\mathbb{H}^{+}(\Gamma,[k])$ cannot be extended for 
a substantially larger class of plumbing graphs (than the $A R$ graphs) - unless one modifies the definition of the combinatorial object $\mathbb{H}^{+}$.

Although the paper is more topological/combinatorial, in some places we emphasize the relevance of the results in the theory of normal surface singularities, especially in the light of [12] and [11].

The reader is invited to read Section 2.5, which contains more details about the guiding problems (and the main results) of the present paper.

The author thanks Péter Ozsváth and Zoltán Szabó for their help, advice and encouragement. The author is partially supported by NSF grant DMS-0304759 and OTKA grants 42769 and 46878.

\section{Preliminaries}

2.1 Plumbing graphs We consider an oriented plumbed 3-manifold $M=$ $M(\Gamma)$ obtained from the graph $\Gamma$ by plumbing construction (with all the edgesigns equal to + ; see [14, Section 8]). We will assume that $M$ is a rational homology sphere. This is equivalent to $\Gamma$ being a disjoint union of trees, all the genera of the corresponding Riemann surfaces involved in the plumbing construction being zero, and the bilinear form $B$ associated with $\Gamma$ (see below) being non-degenerate. Additionally, we will assume that $\Gamma$ is connected and $B$ is negative definite (for motivation see Example 2.1.1, although most of the arguments work for non-connected $\Gamma$ as well). In fact, the plumbing construction provides an oriented $4-$ manifold $\widetilde{X}$ whose oriented boundary $\partial \widetilde{X}$ is exactly $M$. Let $-M$ denote $M$ with the opposite orientation.

The vertices of $\Gamma$ will be indexed by $\mathcal{J}$. Each vertex $j \in \mathcal{J}$ has a decoration $e_{j}$, which is the Euler number of the corresponding disc-bundle (or $S^{1}$-bundle) used in the plumbing construction. Let $L$ be the free $\mathbb{Z}$-module of rank $s:=\# \mathcal{J}$ with a fixed basis $\left\{b_{j}\right\}_{j \in \mathcal{J}}$.

In fact, $\Gamma$ is codified by the bilinear form $B=\left(b_{i}, b_{j}\right)_{i, j \in \mathcal{J}}$, defined by $\left(b_{j}, b_{j}\right)=$ $e_{j}$; and for $i \neq j$ one takes $\left(b_{i}, b_{j}\right)=1$ if the corresponding vertices $i, j$ are connected by an edge in $\Gamma$, otherwise $\left(b_{i}, b_{j}\right)=0$. For any vertex $j \in \mathcal{J}$ of $\Gamma$ we denote by $\delta_{j}$ the number of adjacent vertices.

A cycle $x=\sum_{j} n_{j} b_{j} \in L$ is called effective, denoted by $x \geq 0$, if $n_{j} \geq 0$ for any $j$. We write $x \geq y$ if $x-y \geq 0$. $x>0$ means $x \geq 0$ but $x \neq 0$. We define the support $|x|$ of $x$ by $\bigcup_{j: n_{j} \neq 0}\left\{b_{j}\right\}$. If $x_{i}=\sum_{j} n_{j, i} b_{j}$ for $i=1,2$, then $\min \left\{x_{1}, x_{2}\right\}:=\sum_{j} \min \left\{n_{j, 1}, n_{j, 2}\right\} b_{j}$. We write $x^{2}$ to mean $(x, x)$. 
Set $L^{\prime}=\operatorname{Hom}_{\mathbb{Z}}(L, \mathbb{Z})$, and consider the exact sequence $0 \rightarrow L \stackrel{i}{\rightarrow} L^{\prime} \rightarrow H \rightarrow 0$, where $i(x)=(x, \cdot)$. Then one has the identifications $L \approx H_{2}(\widetilde{X}, \mathbb{Z}), L^{\prime} \approx$ $H_{2}(\widetilde{X}, M, \mathbb{Z})$ and $H \approx H_{1}(M, \mathbb{Z})$.

Sometimes it is convenient to identify the lattice $L^{\prime}$ with a sub-lattice of $L_{\mathbb{Q}}=$ $L \otimes \mathbb{Q}$ : any $\alpha \in L^{\prime}$ is identified with $y_{\alpha} \in L_{\mathbb{Q}}$ which satisfies $\alpha(x)=\left(y_{\alpha}, x\right)$ for any $x \in L$. Here $(\cdot, \cdot)$ is the natural extension of the form $B$ to $L_{\mathbb{Q}}$ (denoted in the same way).

In Sections 10 and 11] we will also use the dual base $\left\{g_{j}\right\}_{j \in \mathcal{J}}$ of $L^{\prime}$ (ie $\left(g_{j}, b_{i}\right)=$ $\delta_{i j}$ for any $\left.i, j \in \mathcal{J}\right)$. If $L^{\prime}$ is identified with a sub-lattice of $L_{\mathbb{Q}}$ then $g_{j}$ is the $j^{\text {th }}$ column of $B^{-1}$. Since $B$ is negative definite, $-g_{j} \geq 0$ for any $j \in \mathcal{J}$.

Clearly, the order $|H|$ of the group $H=H_{1}(M, \mathbb{Z})$ equals $|\operatorname{det}(B)|$.

2.1.1 Example (See [14) Let $M$ be the link of a normal surface singularity $(X, 0)$. Assume that $\widetilde{X} \rightarrow X$ is a good resolution of $(X, 0)$, ie $X$ is a sufficiently small Stein representative of $(X, 0)$ and $\widetilde{X} \rightarrow X$ is a resolution with normal crossing exceptional divisor. Let $\Gamma$ denote the dual resolution graph. Then $\partial \widetilde{X}=M$, and $(\widetilde{X}, \partial \widetilde{X})$ can be identified with the output of the plumbing construction provided by $\Gamma$. (In this case, the elements $b_{j}$ of $L=H_{2}(\widetilde{X}, \mathbb{Z})$ correspond to the classes of the irreducible exceptional divisors.)

Recall, that a plumbed 3-manifold $M$ can be realized as the link of some normal surface singularity if and only if its graph $\Gamma$ is connected and the corresponding form $B$ is negative definite [4].

$2.2 \mathbb{Z}[U]$-modules Later we will use the following notation. Consider the graded $\mathbb{Z}[U]$-module $\mathbb{Z}\left[U, U^{-1}\right]$, and (following [21]) denote by $\mathcal{T}_{0}^{+}$its quotient by the submodule $U \cdot \mathbb{Z}[U]$. This has a grading such that $\operatorname{deg}\left(U^{-d}\right)=2 d$ (for $d \geq$ $0)$. Similarly, for any $n \geq 1$, define the graded module $\mathcal{T}_{0}(n)$ by the quotient of $\mathbb{Z}\left\langle U^{-(n-1)}, U^{-(n-2)}, \ldots, 1, U, \ldots\right\rangle$ by $U \cdot \mathbb{Z}[U]$ (with the same grading). Hence, $\mathcal{T}_{0}(n)$, as a $\mathbb{Z}$-module, is the free $\mathbb{Z}$-module $\mathbb{Z}\left\langle 1, U^{-1}, \ldots, U^{-(n-1)}\right\rangle$ (generated by $\left.1, U^{-1}, \ldots, U^{-(n-1)}\right)$, and has finite $\mathbb{Z}$-rank $n$.

More generally, for any graded $\mathbb{Z}[U]$-module $P$ with $d$-homogeneous elements $P_{d}$, and for any $r \in \mathbb{Q}$, we denote by $P[r]$ the same module graded in such a way that $P[r]_{d+r}=P_{d}$. Then set $\mathcal{T}_{r}^{+}:=\mathcal{T}_{0}^{+}[r]$ and $\mathcal{T}_{r}(n):=\mathcal{T}_{0}(n)[r]$.

2.3 Characteristic elements and $\operatorname{Spin}^{c}$-structures Fix a plumbing graph $\Gamma$ as above. The set of characteristic elements is defined by

$$
\text { Char }=\operatorname{Char}(\Gamma):=\left\{k \in L^{\prime}: k(x)+(x, x) \in 2 \mathbb{Z} \text { for every } x \in L\right\} .
$$


Clearly, for any fixed $k_{0} \in$ Char, Char $=k_{0}+2 L^{\prime}$. There is a natural action of $L$ on Char by $x * k:=k+2 i(x)$ whose orbits are of type $k+2 i(L)$. Obviously, $H$ acts freely and transitively on the set of orbits by $\left[l^{\prime}\right] *(k+2 i(L)):=k+2 l^{\prime}+2 i(L)$ (in particular, they have the same cardinality).

If $\widetilde{X}$ is as in Section 2.1 then the first Chern class (of the determinant line bundle associated with a given $\operatorname{spin}^{c}$-structure) realizes an identification between the $\operatorname{spin}^{c}-$ structures $\operatorname{Spin}^{c}(\widetilde{X})$ on $\widetilde{X}$ and $\operatorname{Char} \subset L^{\prime} \approx H^{2}(\widetilde{X}, \mathbb{Z})$ (see $\underline{3}$, Section 2.4.16]). The restrictions to $M$ defines an identification of the $\operatorname{spin}^{c}$ structures $\operatorname{Spin}^{c}(M)$ of $M$ with the set of orbits of Char modulo $2 L$; and this identification is compatible with the action of $H$ on both sets. In the sequel, we think about $\operatorname{Spin}^{c}(M)$ by this identification, hence any $\operatorname{spin}^{c}$-structure of $M$ will be represented by an orbit $[k]:=k+2 i(L) \subset$ Char.

The $\operatorname{spin}^{c}$-structures $\operatorname{Spin}^{c}(M)$ and $\operatorname{Spin}^{c}(-M)$ are canonically identified.

2.4 Invariants of the 3-manifold $M$ In this note we will focus on the following set of invariants.

2.4.1 According to Turaev 24, a choice of a $\operatorname{spin}^{c}$-structure on $M$ is equivalent to a choice of an Euler structure. For every $\operatorname{spin}^{c}$-structure $[k]$, he defines the sign-refined Reidemeister-Turaev torsion

$$
\mathcal{T}_{M,[k]}=\sum_{h \in H} \mathcal{T}_{M,[k]}(h) h \in \mathbb{Q}[H]
$$

determined by the Euler structure associated with $[k]$ (see [24]). It is convenient to think about $\mathcal{T}_{M,[k]}$ as a function $H \rightarrow \mathbb{Q}$ given by $h \mapsto \mathcal{T}_{M,[k]}(h)$. We will be basically interested in $\mathcal{T}_{M,[k]}(1)$ where 1 denotes the neutral element of the group $H$ (with the multiplicative notation).

For plumbed 3-manifold $M$ (as in Section 2.1), 12, Section 5.7] provides a combinatorial formula for $\mathcal{T}_{M,[k]}(1)$ in terms of $\Gamma$ (involving regularized FourierDedekind sums).

2.4.2 We will use the notation $\lambda(M)$ for the Casson-Walker invariant of $M$ (normalized as in [7, Section 4.7]). For plumbed manifolds $M$, it has a combinatorial formula from $\Gamma$ (provided by A. Raţiu in his dissertation; and it can be deduced from the surgery formulas of [7] as well; see also [12, Section 5.3]):

$$
-\frac{24}{|H|} \lambda(M)=\sum_{j} e_{j}+3 s+\sum_{j}\left(2-\delta_{j}\right)\left(B^{-1}\right)_{j j}
$$


2.4.3 If $M$ is a plumbed 3-manifold as in Section 2.1 it has a canonical characteristic element $K$ defined by $K\left(b_{j}\right)=-e_{j}-2$ for any $j$ (see also 5.1.1 for more comments about these "adjunction formulas"). The rational number $K^{2}+s$ does not depend on the (negative definite) plumbing graph, it is an invariant of $M$. It can be computed from $\Gamma$ as follows (see [12, Section 5.2]):

$$
K^{2}+s=\sum_{j} e_{j}+3 s+2+\sum_{i, j}\left(2-\delta_{i}\right)\left(2-\delta_{j}\right)\left(B^{-1}\right)_{i j} .
$$

2.4.4 The Heegaard Floer homology $\mathrm{HF}^{+}(M,[k])$ For any oriented rational homology 3-sphere $M$ and $[k] \in \operatorname{Spin}^{c}(M)$, the Ozsváth-Szabó $\mathbb{Z}[U]-$ module (or, the absolutely graded Heegaard Floer homology) $\mathrm{HF}^{+}(\mathrm{M},[k])$ was introduced in [17] (see also the long list of recent articles of Ozsváth and Szabó). This has a $\mathbb{Q}$-grading compatible with the $\mathbb{Z}[U]$-action, where $\operatorname{deg}(U)=-2$.

Additionally, $H F^{+}(M,[k])$ has an (absolute) $\mathbb{Z}_{2}$-grading; $H F_{\text {even }}^{+}(M,[k])$, respectively $H F_{\text {odd }}^{+}(M,[k])$ denote the part of $H_{F}^{+}(M,[k])$ with the corresponding parity.

By the general theory, for any $\operatorname{spin}^{c}$-structure $[k]$ and $M$ as above, one has a graded $\mathbb{Z}[U]$-module isomorphism

$$
H F^{+}(M,[k])=\mathcal{T}_{d(M,[k])}^{+} \oplus H F_{\text {red }}^{+}(M,[k]),
$$

where $H F_{\text {red }}^{+}$has a finite $\mathbb{Z}$-rank and an induced (absolute) $\mathbb{Z}_{2}$-grading (and $d(M,[k])$ can also be defined via this isomorphism).

From the above data one can extract two crucial numerical invariants: $d(M,[k])$ and

$$
\chi\left(H F^{+}(M,[k])\right):=\operatorname{rank}_{\mathbb{Z}} H F_{\text {red,even }}^{+}(M,[k])-\operatorname{rank}_{\mathbb{Z}} H F_{\text {red,odd }}^{+}(M,[k]) .
$$

With respect to the change of orientation they behave as follows:

$$
d(M,[k])=-d(-M,[k]) \text { and } \chi\left(H F^{+}(M,[k])\right)=-\chi\left(H F^{+}(-M,[k])\right) .
$$

Notice that one can recover $H F^{+}(M,[k])$ from $H F^{+}(-M,[k])$ by a standard procedure described by Ozsváth and Szabó.

2.4.5 The combinatorial module $\mathbb{H}^{+}(\Gamma)$ In the case of a plumbed 3manifold $M$ associated with the graph $\Gamma$, one would like to obtain a completely combinatorial description of $H^{+}(-M,[k])$ from $\Gamma$. In [21, Ozsváth and Szabó introduced the following combinatorial graded $\mathbb{Z}[U]$-module as a candidate. 
Let $\mathbb{H}^{+}(\Gamma)$ be the set of those finitely supported functions $\phi$ : Char $\rightarrow \mathcal{T}_{0}^{+}$ which satisfy the following property. For any characteristic element $k$ and base vector $b_{j}$ write $2 n=k\left(b_{j}\right)+\left(b_{j}, b_{j}\right)$. Then if $n \geq 0$ then one requires

$$
U^{n} \cdot \phi\left(k+2 i\left(b_{j}\right)\right)=\phi(k)
$$

while if $n \leq 0$, then

$$
\phi\left(k+2 i\left(b_{j}\right)\right)=U^{-n} \cdot \phi(k) .
$$

For any $\operatorname{spin}^{c}$-structure $[k]=k+2 i(L)$, let $\mathbb{H}^{+}(\Gamma,[k])$ be the subset of those maps $\phi$ which are supported on $k+2 i(L)$. Then one has a direct sum splitting $\mathbb{H}^{+}(\Gamma)=\bigoplus_{[k]} \mathbb{H}^{+}(\Gamma,[k])$.

On $\mathbb{H}^{+}(\Gamma)$ one defines the following (rational) grading. One says that a map $\phi \in \mathbb{H}^{+}(\Gamma)$ is homogeneous of degree $d \in \mathbb{Q}$ if for each characteristic vector $k$ with $\phi(k) \neq 0, \phi(k) \in \mathcal{T}_{0}^{+}$is a homogeneous element with

$$
\operatorname{deg}(\phi(k))-\frac{k^{2}+s}{4}=d .
$$

Ozsváth and Szabó in [21] proved the following theorem.

2.4.6 Theorem Assume that $\Gamma$ has at most one vertex $j \in \mathcal{J}$ where the inequality $-e_{j} \geq \delta_{j}$ fails. Then for any $\operatorname{spin}^{c}$-structure $[k], H F_{\text {odd }}^{+}(-M,[k])=$ 0 and $H F_{\text {even }}^{+}(-M,[k])=\mathbb{H}^{+}(\Gamma,[k])$.

2.5 The main guiding problems of the article We summarize in short those conjectures and problems which have determined our interest in this subject. This is an interesting mixture of the topology of 3 -manifolds and theory of normal surface singularities.

2.5.1 Problem I First of all, as we already mentioned, for any plumbed manifold $M$ one wishes to determine $\mathrm{HF}^{+}(M,[k])$ in a combinatorial way from $\Gamma$.

Our goal (realized in Section 8.3) is to extend the isomorphism 2.4.6 for a larger class of plumbing graphs, namely for the $A R$ graphs (introduced in section 8). This class includes all the links of rational and elliptic singularities, contains the class considered by Ozsváth and Szabó in Theorem 2.4.6, in particular, all the Seifert manifolds with negative orbifold Euler number. (Examples show that the statement of Theorem 2.4.6] cannot be extended for a substantially larger class of graphs; see Section 8.4) 
2.5.2 Problem II Even though $\mathbb{H}^{+}(\Gamma,[k])$ is combinatorial, its computation is not trivial at all. In the body of the paper we provide precise algorithm for this computation (valid for any $A R$ graph).

The main idea comes from the technique of computation sequences used in singularity theory (see [5] and [6]). In fact, as a starting invariant, we will construct a graded root $R_{k}$ for any plumbing graph $\Gamma$ and for any characteristic element $k$. Surprisingly, this object is able to guide (and connect) two very different theories. First, from the point of view of singularity theory, it seems that it is the right object which controls the hierarchy and classification of surface singularities. For example, it characterizes nicely the rational and elliptic singularities (see Section 6). Also, it provides optimal topological upper bounds for some analytic invariants (see Problem IV below and Section 9.6). On the other hand, from $R_{k}$ one can read easily $\mathbb{H}^{+}(\Gamma,[k])$.

Our discussion runs on three levels: we determine for any $A R$ graph the graded root $R_{k}$, which determines automatically the combinatorial Ozsváth-Szabó graded $\mathbb{Z}[U]$-modules $\mathbb{H}^{+}(\Gamma,[k])$, and finally we focus on the numerical invariants $d(M,[k])$ and $\chi\left(H F^{+}(M,[k])\right.$ as well.

One of the surprising byproducts of the discussion is Theorem 6.3 which shows that the links of rational singularities correspond exactly to the $L$-spaces in the sense of Ozsváth-Szabó.

2.5.3 Problem III One of the goals of Ozsváth-Szabó homology is to substitute the (modified) Seiberg-Witten topological invariant of $(M,[k])$. More precisely, it provides the numerical invariant

$$
\mathbf{s w}_{M,[k]}^{O S z}:=\chi\left(H F^{+}(M,[k])\right)-\frac{d(M,[k])}{2}
$$

as a candidate for the Seiberg-Witten invariant.

On the other hand, the sign-refined Reidemeister-Turaev torsion together with the Casson-Walker invariant also provides a candidate for the Seiberg-Witten invariant (see also [16]):

$$
\mathbf{s w}_{M,[k]}^{T C W}:=-\mathcal{T}_{M,[k]}(1)+\frac{\lambda(M)}{|H|},
$$

where $|H|$ is the order of $H$.

Our goal is to investigate the identity of these two candidates. Notice that in the presence of $H F_{\text {red,odd }}^{+}(-M,[k])=0$, this conjectured identity reads as

$$
\mathcal{T}_{M,[k]}(1)-\frac{\lambda(M)}{|H|}=\operatorname{rank}_{\mathbb{Z}} H F_{\text {red }}^{+}(-M,[k])-\frac{d(-M,[k])}{2} .
$$


Recall that if $M$ is an integral homology sphere (ie $H=0$ ) then this identity holds by [20, Section 1.3].

Since for $A R$ graphs the right hand side of the identity can be determined from $\mathbb{H}^{+}(\Gamma,[k])$, the above identity becomes a purely combinatorial property of $\Gamma$. In the body of the paper we verify it for any lens space and Seifert manifold (and any $\operatorname{spin}^{c}$-structure).

2.5.4 Problem IV In 12, L. Nicolaescu and the author stated the following conjecture. Assume that $(X, 0)$ is a normal surface singularity whose link $M$ is a rational homology sphere. Let $p_{g}$ be the geometric genus of $(X, 0)$ (ie $\operatorname{dim}_{\mathbb{C}} H^{1}\left(\tilde{X}, \mathcal{O}_{\tilde{X}}\right)$, where $\tilde{X}$ is a resolution of $\left.X\right)$. Then the analytic invariant $p_{g}$ has the following "optimal" topological upper bound:

$$
p_{g} \leq \mathcal{T}_{M,[K]}(1)-\frac{\lambda(M)}{|H|}-\frac{K^{2}+s}{8}
$$

Moreover, if $(X, 0)$ is $\mathbb{Q}$-Gorenstein, then above one has equality.

Notice that in the presence of $H F_{\text {red,odd }}^{+}(-M,[K])=0$, and using the principle in Problem III above, this inequality can be transformed into another conjectured inequality

$$
p_{g} \leq \operatorname{rank}_{\mathbb{Z}}\left(H F_{\text {red }}^{+}(-M,[K])\right)+\frac{d(M,[K])}{2}-\frac{K^{2}+s}{8}
$$

which conjecturally becomes equality for $\mathbb{Q}$-Gorenstein singularities.

The point is that the computation algorithm of $\mathbb{H}^{+}(\Gamma,[K])$ of the present article will automatically provide this inequality for any singularity with $A R$-resolution graph. Moreover, we also prove the identity for rational and elliptic Gorenstein singularities and all singularities which admit a good $\mathbb{C}^{*}$-action (all of these are $\mathbb{Q}$-Gorenstein).

In fact, in Corollary 9.6, we extend the above inequality for any $\operatorname{spin}^{c}$ structure and any $A R$ graph. In this way, the (candidates for the) Seiberg-Witten invariants (provided by the Heegaard Floer theory) provide a (conjecturally optimal) topological upper bound for $h^{1}(\mathcal{L})$, where $\mathcal{L}$ is a holomorphic line bundle on a resolution of $(X, 0)$.

For the extension of the conjecture [12 to arbitrary $\operatorname{spin}^{c}$ structures, see [11. For different definitions regarding surface singularities, the reader is invited to consult [10].

After the first version of the present article was completed, the author realized that the above inequality - and equality for Gorenstein singularities - is not true for non- $A R$ singularities; for details see [8]. 


\section{Graded roots}

3.1 Preliminary remarks We recall that the Ozsváth-Szabó $\mathbb{Z}[U]$-module $\mathbb{H}^{+}(\Gamma,[k])$ is defined in a combinatorial way from the plumbing graph $\Gamma$. Our goal is to define an intermediate object, a graded root $R_{k}$ associated with $\Gamma$ (and a characteristic element $k$ ). This will contain all the needed information to determine the homological object $\mathbb{H}^{+}$, but it preserves also some additional, more subtle topological information about $\Gamma$ (or, about $M$ ).

In this section we give the definition and first properties of the (abstract) graded roots. The next section contains the construction of the graded roots $R_{k}$ from the plumbing graphs $\Gamma$. (Although both $\Gamma$ (the plumbing graph) and the constructed graded root $R_{k}$ are "connected trees", they serve rather different roles. For example, the edges of $\Gamma$ codify the corresponding gluings in the plumbing, while the edges of $R_{k}$ codify the $\mathbb{Z}[U]$-action. We hope the terminology will not create any confusion.)

3.2 Definitions (1) Let $R$ be an infinite tree with vertices $\mathcal{V}$ and edges $\mathcal{E}$. We denote by $[u, v]$ the edge with end-points $u$ and $v$. We say that $R$ is a graded root with grading $\chi: \mathcal{V} \rightarrow \mathbb{Z}$ if

(a) $\chi(u)-\chi(v)= \pm 1$ for any $[u, v] \in \mathcal{E}$;

(b) $\chi(u)>\min \{\chi(v), \chi(w)\}$ for any $[u, v],[u, w] \in \mathcal{E}, v \neq w$;

(c) $\quad \chi$ is bounded below, $\chi^{-1}(k)$ is finite for any $k \in \mathbb{Z}$, and $\# \chi^{-1}(k)=1$ if $k$ is sufficiently large.

(2) We say that $v \in \mathcal{V}$ is a local minimum point of the graded root $(R, \chi)$ if $\chi(v)<\chi(w)$ for any edge $[v, w]$.

(3) If $(R, \chi)$ is a graded root, and $r \in \mathbb{Z}$, then we denote by $(R, \chi)[r]$ the same $R$ with the new grading $\chi[r](v):=\chi(v)+r$. (This can be generalized for any $r \in \mathbb{Q}$ as well.)

3.3 Notation and remarks (1) For a vertex $v$ set $\delta_{v}:=\#\{[v, w] \in \mathcal{E}\}$. One can verify that the set of vertices $\mathcal{V}_{1}:=\left\{v \in \mathcal{V}: \delta_{v}=1\right\}$ are exactly the local minimum points of $\chi$, and $\# \mathcal{V}_{1}<\infty$.

(2) A geodesic path connecting two vertices is monotone if $\chi$ restricted to the set of vertices on the path is strict monotone. If a vertex $v$ can be connected by another vertex $w$ by a monotone geodesic and $\chi(v)>\chi(w)$, then we say that $v$ dominates $w$, and we write $v \succ w$. $\succ$ is an ordering of $\mathcal{V}$. For any pair $v, w \in \mathcal{V}$ there is a unique $\succ-$ minimal vertex $\sup (v, w)$ which dominates both. 
3.4 Examples (1) For any integer $n \in \mathbb{Z}$, let $R_{n}$ be the tree with $\mathcal{V}=$ $\left\{v^{k}\right\}_{k \geq n}$ and $\mathcal{E}=\left\{\left[v^{k}, v^{k+1}\right]\right\}_{k \geq n}$. The grading is $\chi\left(v^{k}\right)=k$.

(2) Let $I$ be a finite index set. For each $i \in I$ fix an integer $n_{i} \in \mathbb{Z}$; and for each pair $i, j \in I$ fix $n_{i j}=n_{j i} \in \mathbb{Z}$ with the properties

(i) $n_{i i}=n_{i}$;

(ii) $n_{i j} \geq \max \left\{n_{i}, n_{j}\right\}$; and

(iii) $n_{j k} \leq \max \left\{n_{i j}, n_{i k}\right\}$

for any $i, j, k \in I$.

For any $i \in I$ consider $R_{i}:=R_{n_{i}}$ with vertices $\left\{v_{i}^{k}\right\}$ and edges $\left\{\left[v_{i}^{k}, v_{i}^{k+1}\right]\right\}$, $\left(k \geq n_{i}\right)$. In the disjoint union $\coprod_{i} R_{i}$, for any pair $(i, j)$, identify $v_{i}^{k}$ and $v_{j}^{k}$, respectively $\left[v_{i}^{k}, v_{i}^{k+1}\right]$ and $\left[v_{j}^{k}, v_{j}^{k+1}\right]$, whenever $k \geq n_{i j}$. Write $\bar{v}_{i}^{k}$ for the class of $v_{i}^{k}$. Then $\coprod_{i} R_{i} / \sim$ is a graded root with $\chi\left(\bar{v}_{i}^{k}\right)=k$. It will be denoted by $R=R\left(\left\{n_{i}\right\},\left\{n_{i j}\right\}\right)$.

Clearly $\mathcal{V}_{1}(R)$ is a subset of $\left\{\bar{v}_{i}^{n_{i}}\right\}_{i \in I}$, and this last set can be identified with $I$. $\mathcal{V}_{1}(R)=I$ if in (ii) all the inequalities are strict. Otherwise all the indices $I \backslash \mathcal{V}_{1}(R)$ are superfluous, ie the corresponding $R_{i}$ 's produce no additional vertices.

In fact, any graded root $\left(R^{\prime}, \chi^{\prime}\right)$ is isomorphic (in a natural sense) with some $R\left(\left\{n_{i}\right\},\left\{n_{i j}\right\}\right)$. Indeed, set $I:=\mathcal{V}_{1}\left(R^{\prime}\right), n_{v}:=\chi^{\prime}(v)$ and $n_{u v}:=\chi^{\prime}(\sup (u, v))$ for $u, v \in I$.

(3) Any map $\tau:\{0,1, \ldots, l\} \rightarrow \mathbb{Z}$ produces starting data for construction (2). Indeed, set $I=\{0, \ldots, l\}, n_{i}:=\tau(i)$ for $i \in I$, and $n_{i j}:=\max \left\{n_{k}: i \leq k \leq j\right\}$ for $i \leq j$. Then $\coprod_{i} R_{i} / \sim$ constructed in (2) using this data will be denoted by $\left(R_{\tau}, \chi_{\tau}\right)$.

For example, for $l=4$, take for the values of $\tau:-3,-1,-2,0$ and -2 (respectively $-3,0,-2,-1$ and -2$)$. Then the two graded roots are:
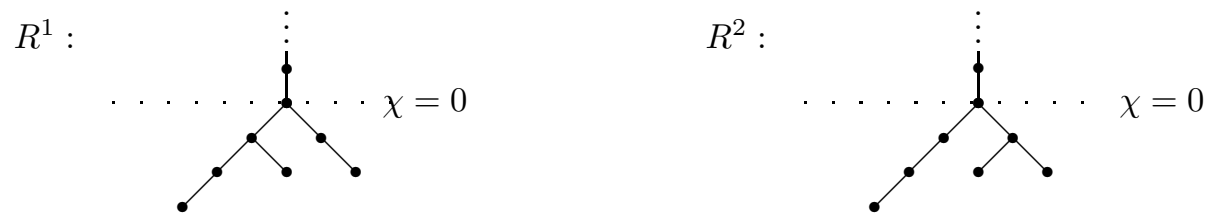

In fact, we can even start with the infinite index set $I=\mathbb{N}$, and a function $\tau: \mathbb{N} \rightarrow \mathbb{Z}$, provided that we assure that starting from a bound $l$, all the contributions $R_{i}$ with $i>l$ are superfluous. This happens, for example, if, for some $l, \tau(i+1) \geq \tau(i)$ for any $i \geq l$. 
3.5 Definition: the associated $\mathbb{Z}[U]-$ modules For a graded root $(R, \chi)$, let $\mathbb{H}(R, \chi)$ (shortly $\mathbb{H}(R)$ ) be the set of functions $\phi: \mathcal{V} \rightarrow \mathcal{T}_{0}^{+}$with the following property: whenever $[v, w] \in \mathcal{E}$ with $\chi(v)<\chi(w)$, then

$$
U \cdot \phi(v)=\phi(w) .
$$

Or, equivalently, for any $w \succ v$ one requires

$$
U^{\chi(w)-\chi(v)} \cdot \phi(v)=\phi(w) .
$$

Clearly $\mathbb{H}(R)$ is a $\mathbb{Z}[U]$-module via $(U \phi)(v)=U \cdot \phi(v)$. Moreover, $\mathbb{H}(R)$ has a grading: $\phi \in \mathbb{H}(R)$ is homogeneous of degree $d \in \mathbb{Z}$ if for each $v \in \mathcal{V}$ with $\phi(v) \neq 0, \phi(v) \in \mathcal{T}_{0}^{+}$is homogeneous of degree $d-2 \chi(v)$. Notice that in $(*)$ one has $2 \chi(v)+\operatorname{deg} \phi(v)=2 \chi(w)+\operatorname{deg} \phi(w)$, hence $d$ is well-defined.

Notice that any $\phi$ as above is automatically finitely supported.

3.5.1 From the definitions, it is clear that $\mathbb{H}((R, \chi)[r])=\mathbb{H}(R, \chi)[2 r]$ for any $r \in \mathbb{Z}$.

3.5.2 Proposition Let $(R, \chi)$ be a graded root. Set $I:=\left\{v \in \mathcal{V}: \delta_{v}=1\right\}$, and we order the set $I$ as follows. The first element $v_{1}$ is an arbitrary vertex with $\chi\left(v_{1}\right)=\min _{v} \chi(v)$. If $v_{1}, \ldots, v_{k}$ is already determined, and $J:=$ $\left\{v_{1}, \ldots, v_{k}\right\} \varsubsetneqq I$, then let $v_{k+1}$ be an arbitrary vertex in $I \backslash J$ with $\chi\left(v_{k+1}\right)=$ $\min _{v \in I \backslash J} \chi(v)$. Let $w_{k+1} \in \mathcal{V}$ be the unique $\succ-$ minimal vertex of $R$ which dominates both $v_{k+1}$, and at least one vertex from $J$. Then one has the following isomorphism of $\mathbb{Z}[U]$-modules:

$$
\mathbb{H}(R, \chi)=\mathcal{T}_{2 \chi\left(v_{1}\right)}^{+} \oplus \bigoplus_{k \geq 2} \mathcal{T}_{2 \chi\left(v_{k}\right)}\left(\chi\left(w_{k}\right)-\chi\left(v_{k}\right)\right)
$$

In particular, with the notation $m:=\min _{v} \chi(v)$ and

$$
\mathbb{H}_{\text {red }}(R, \chi):=\bigoplus_{k \geq 2} \mathcal{T}_{2 \chi\left(v_{k}\right)}\left(\chi\left(w_{k}\right)-\chi\left(v_{k}\right)\right),
$$

one has a canonical direct sum decomposition

$$
\mathbb{H}(R, \chi)=\mathcal{T}_{2 m}^{+} \oplus \mathbb{H}_{\text {red }}(R, \chi)
$$

of graded $\mathbb{Z}[U]$-modules. The $\mathbb{Z}[U]$-module $\mathbb{H}_{\text {red }}(R)$ has finite $\mathbb{Z}$-rank, with $\mathbb{H}_{\text {red }}(R)=0$ if and only if $\# I=1$ and $R=R_{\min \chi}$.

Proof The proof is elementary, and is left to the reader. 


\subsection{Examples (a) $\mathbb{H}\left(R_{n}\right)=\mathcal{T}_{2 n}$.}

(b) The graded roots $R^{1}$ and $R^{2}$ constructed in Example 3.4 (3) are not isomorphic, but their $\mathbb{Z}[U]$-modules are: $\mathbb{H}\left(R^{1}\right)=\mathbb{H}\left(R^{2}\right)=\mathcal{T}_{-6}^{+} \oplus \mathcal{T}_{-4}(1) \oplus \mathcal{T}_{-4}(2)$. Hence, in general, a graded root carries more information than its $\mathbb{Z}[U]$-module.

3.7 Corollary Let $\left(R_{\tau}, \chi_{\tau}\right)$ be a graded root associated with some function $\tau: \mathbb{N} \rightarrow \mathbb{Z}$ (see Example 3.4(3)) which satisfies $\tau(1)>\tau(0)$. Then the $\mathbb{Z}$-rank of $\mathbb{H}_{\text {red }}\left(R_{\tau}, \chi_{\tau}\right)$ is

$$
\operatorname{rank}_{\mathbb{Z}} \mathbb{H}_{\text {red }}\left(R_{\tau}\right)=-\tau(0)+\min _{i \geq 0} \tau(i)+\sum_{i \geq 0} \max \{\tau(i)-\tau(i+1), 0\} .
$$

The summand $\mathcal{T}_{2 m}^{+}$of $\mathbb{H}\left(T_{\tau}, \chi_{\tau}\right)$ has index $m=\min _{i \geq 0} \tau(i)=\min _{v} \chi_{\tau}(v)$.

Proof Use induction over $l($ where $\tau:\{0, \ldots, l\} \rightarrow \mathbb{Z})$.

\section{Graded roots associated with plumbing graphs}

4.1 Fix a connected plumbing graph $\Gamma$ whose bilinear form is negative definite (see Section 2.1). In this section we will construct a graded root $\left(R_{k}, \chi_{k}\right)$ associated with any characteristic element $k$.

4.2 The construction of $\left(R_{k}, \chi_{k}\right)$ Fix $k \in$ Char and define $\chi_{k}: L \rightarrow \mathbb{Z}$ by

$$
\chi_{k}(x):=-(k(x)+(x, x)) / 2 .
$$

For any $n \in \mathbb{Z}$, we define a finite 1 -dimensional simplicial complex $\bar{L}_{k, \leq n}$ as follows. Its 0 -skeleton is $L_{k, \leq n}:=\left\{x \in L: \chi_{k}(x) \leq n\right\}$. For each $x$ and $j \in \mathcal{J}$ with $x, x+b_{j} \in L_{k, \leq n}$, we consider a unique 1 -simplex with endpoints at $x$ and $x+b_{j}\left(\mathrm{eg}\right.$, the segment $\left[x, x+b_{j}\right]$ in $\left.L \otimes \mathbb{R}\right)$. We denote the set of connected components of $\bar{L}_{k, \leq n}$ by $\pi_{0}\left(\bar{L}_{k, \leq n}\right)$. For any $v \in \pi_{0}\left(\bar{L}_{k, \leq n}\right)$, let $C_{v}$ be the corresponding connected component of $\bar{L}_{k, \leq n}$.

Next, we define the graded root $\left(R_{k}, \chi_{k}\right)$ as follows. The vertices $\mathcal{V}\left(R_{k}\right)$ are $\bigcup_{n \in \mathbb{Z}} \pi_{0}\left(\bar{L}_{k, \leq n}\right)$. The grading $\mathcal{V}\left(R_{k}\right) \rightarrow \mathbb{Z}$, which we still denote by $\chi_{k}$, is $\chi_{k} \mid \pi_{0}\left(\bar{L}_{k, \leq n}\right)=n$.

If $v_{n} \in \pi_{0}\left(\bar{L}_{k, \leq n}\right)$, and $v_{n+1} \in \pi_{0}\left(\bar{L}_{k, \leq n+1}\right)$, and $C_{v_{n}} \subset C_{v_{n+1}}$, then $\left[v_{n}, v_{n+1}\right]$ is an edge of $R_{k}$. All the edges $\mathcal{E}\left(R_{k}\right)$ of $R_{k}$ are obtained in this way.

4.3 Proposition For any $k \in$ Char, $\left(R_{k}, \chi_{k}\right)$ is a graded root. 
Proof Clearly, $\# \mathcal{V}\left(R_{k}\right)$ is infinite. Notice that the validity of Definition 3.2(1) part (b) guarantees that $R_{k}$ has no closed cycles. Indeed, in the presence of a cycle $v_{1}, v_{2}, \ldots, v_{n}, v_{1}$ in $R_{k}, \chi_{k}$ restricted to the set $\left\{v_{1}, \ldots, v_{n}\right\}$ would have a local minimum which would contradict Definition 3.2(1) part (b). Hence, it is enough to verify properties Definition [3.2(1) parts (a)-(c). Property (a) is clear. With the notation of (b), assume that (b) is not true for some $u, v, w$, ie $\chi_{k}(u)<$ $\min \left\{\chi_{k}(v), \chi_{k}(w)\right\}$. This would imply $C_{u} \subset C_{v} \cap C_{w}$. But this is impossible: two different connected components of a space cannot simultaneously contain a non-empty connected subset. The first two statements of (c) follow from the fact that $B$ is negative definite. Finally, we have to show that $\bar{L}_{k, \leq n}$ is connected for $n$ sufficiently large. Set

$$
\mathcal{Y}:=\left\{x \in L: \chi_{k}(x) \leq \chi_{k}\left(x+\epsilon_{j} b_{j}\right) \text { for all } j \in \mathcal{J}, \text { and } \epsilon_{j}= \pm 1\right\} .
$$

Then, using the definition of $\chi_{k}, \mathcal{Y}$ is

$$
\left\{x \in L:-\chi_{k}\left(-b_{j}\right) \leq\left(x, b_{j}\right) \leq \chi_{k}\left(b_{j}\right) \text { for all } j \in \mathcal{J}\right\} .
$$

Hence $\mathcal{Y}$ is finite, and obviously non-empty since $\chi_{k}$ has a global minimum (which is in $\mathcal{Y}$ ). Fix $y_{*} \in \mathcal{Y}$, and for each $y \in \mathcal{Y} \backslash\left\{y_{*}\right\}$ fix a path which connects $y_{*}$ and $y$. By this we mean a sequence of elements $x_{1}, x_{2}, \ldots, x_{t} \in L$ so that $x_{1}=y_{*}, x_{t}=y$, and $x_{l+1}=x_{l}+\epsilon_{l} b_{j(l)}$ for some $\epsilon_{l} \in\{+1,-1\}$. Let $n_{0}$ be the maximum of all the values of type $\chi_{k}\left(x_{l}\right)$, where $x_{l}$ is an element on one of the above paths connecting $y_{*}$ with some $y$. Then $\bar{L}_{k, \leq n}$ is connected provided that $n \geq n_{0}$.

Hence, for any $k \in$ Char we get a graded root $\left(R_{k}, \chi_{k}\right)$. Some of these graded roots are not very different. Indeed, assume that $k$ and $k^{\prime}$ determine the same $\operatorname{spin}^{c}$ structure, hence $k^{\prime}=k+2 l$ for some $l \in L$. Then $\chi_{k^{\prime}}(x-l)=\chi_{k}(x)-\chi_{k}(l)$ for any $x \in L$. This means that the transformation $x \mapsto x^{\prime}:=x-l$ realizes an identification of $\bar{L}_{k, \leq n}$ and $\bar{L}_{k^{\prime}, \leq n-\chi_{k}(l)}$. Hence, we get:

4.4 Proposition If $k^{\prime}=k+2 l$ for some $l \in L$, then

$$
\left(R_{k^{\prime}}, \chi_{k^{\prime}}\right)=\left(R_{k}, \chi_{k}\right)\left[-\chi_{k}(l)\right] \text {. }
$$

In fact, there is an easy way to choose a graded root from the multitude $\left\{\left(R_{k}, \chi_{k}\right)\right\}_{k \in[k]}$. For any $k \in$ Char we define

$$
m_{k}:=\frac{k^{2}-\max _{k^{\prime} \in[k]}\left(k^{\prime}\right)^{2}}{8} \leq 0 .
$$

Clearly, $m_{k}$ is an integer (see the proof of the next lemma). Set also $M_{[k]}:=$ $\left\{k \in[k]: m_{k}=0\right\}$. 
4.5 Lemma Fix a $\operatorname{spin}^{c}$ structure $[k]$. Then $k_{0} \in M_{[k]}$ if and only if $-\chi_{k_{0}}(l) \leq$ 0 for any $l \in L$. Moreover, if $k_{0}$ and $k_{0}+2 l \in M_{[k]}$, then $-\chi_{k_{0}}(l)=0$.

Proof Write $\left(k_{0}+2 l, k_{0}+2 l\right)=\left(k_{0}, k_{0}\right)-8 \chi_{k_{0}}(l)$.

4.5.1 In particular, using Proposition 4.4 and Lemma 4.5. for any fixed $\operatorname{spin}^{c}$ structure $[k]$, any choice of $k_{0} \in M_{[k]}$ provides the same graded root $\left(R_{k_{0}}, \chi_{k_{0}}\right)$, which will be denoted by $\left(R_{[k]}, \chi_{[k]}\right)$. Moreover, for any $k \in[k]$

$$
\left(R_{k}, \chi_{k}\right)=\left(R_{[k]}, \chi_{[k]}\right)\left[m_{k}\right],
$$

where $m_{k}$ is defined in (11). The notation is compatible with Proposition 3.5 .2

$$
m_{k}=\min \chi_{k}
$$

Indeed, if $k_{0} \in M_{[k]}$, then $m_{k_{0}}=0$ from (11), and $\min \chi_{k_{0}}=0$ from Proposition 4.5. Then (3) follows from (2). The relations (11) and (3) can be summarized in

$$
k^{2}-8 \min \chi_{k}=\max _{k^{\prime} \in[k]}\left(k^{\prime}\right)^{2} .
$$

Clearly, many different plumbing graphs can provide the same 3-manifold $M$. But all these plumbing graphs can be connected by each other by a finite sequence of blowups/downs (-1)-vertices, whose number of incident edges is $\leq 2$. (This fact follows from the existence of an unique minimal resolution graph of a normal surface singularity.)

The next result shows that the set $\left\{\left(R_{[k]}, \chi_{[k]}\right)\right\}_{[k]}$ depends only on $M$.

4.6 Proposition The set $\left(R_{[k]}, \chi_{[k]}\right.$ ) (where $[k]$ runs over the $\operatorname{spin}^{c}$ structures of $M$ ) depends only on $M$ and is independent of the choice of the (negative definite) plumbing graph $\Gamma$ which provides $M$. (See also [21, Section 2.5].)

Proof By the above remark, it is enough to prove that the above set of graded roots is not modified during a blowup. First we assume that $\Gamma^{\prime}$ is obtained from $\Gamma$ by "blowing up a smooth point of the Riemann surface" which in the plumbing construction corresponds to a fixed index $j_{0} \in \mathcal{J}$. More precisely, $\Gamma^{\prime}$ denotes a graph with one more vertex and one more edge than $\Gamma$ : we glue to the vertex $j_{0}$ by the new edge the new vertex with decoration -1 , the decoration of $b_{j_{0}}$ is modified from $e_{j_{0}}$ into $e_{j_{0}}-1$, and we keep all the other decorations. We will use the notation $L(\Gamma), L\left(\Gamma^{\prime}\right), L^{\prime}(\Gamma), L^{\prime}\left(\Gamma^{\prime}\right), B, B^{\prime}$ for the corresponding invariants. Set $b_{\text {new }}$ for the new base element in $L\left(\Gamma^{\prime}\right)$ (with $\left.B^{\prime}\left(b_{\text {new }}, b_{\text {new }}\right)=-1\right)$. The following facts can be verified: 
- Consider the maps $\pi_{*}: L\left(\Gamma^{\prime}\right) \rightarrow L(\Gamma)$ defined by

$$
\pi_{*}\left(\sum x_{j} b_{j}+x_{\text {new }} b_{\text {new }}\right)=\sum x_{j} b_{j},
$$

and $\pi^{*}: L(\Gamma) \rightarrow L\left(\Gamma^{\prime}\right)$ defined by

$$
\pi^{*}\left(\sum x_{j} b_{j}\right)=\sum x_{j} b_{j}+x_{j_{0}} b_{\text {new }} .
$$

Then $B^{\prime}\left(\pi^{*} x, x^{\prime}\right)=B\left(x, \pi_{*} x^{\prime}\right)$. This shows that $B^{\prime}\left(\pi^{*} x, \pi^{*} y\right)=B(x, y)$ and $B^{\prime}\left(\pi^{*} x, b_{\text {new }}\right)=0$ for any $x, y \in L(\Gamma)$.

- Identify (for both graphs) $L^{\prime}$ with a sub-lattice of $L_{\mathbb{Q}}$ (see Section 2.11). Then consider the (nonlinear) map $c: L^{\prime}(\Gamma) \rightarrow L^{\prime}\left(\Gamma^{\prime}\right)$ defined by

$$
\sum r_{j} b_{j} \mapsto \sum r_{j} b_{j}+\left(r_{j_{0}}+1\right) b_{\text {new }}
$$

where $r_{j} \in \mathbb{Q}$, or equivalently, $c\left(l^{\prime}\right):=\pi^{*}\left(l^{\prime}\right)+b_{\text {new }}$. Then $c(\operatorname{Char}(\Gamma)) \subset$ $\operatorname{Char}\left(\Gamma^{\prime}\right)$ and furthermore $c$ induces an isomorphism between the orbit spaces $\operatorname{Char}(\Gamma) / 2 L(\Gamma)$ and $\operatorname{Char}\left(\Gamma^{\prime}\right) / 2 L\left(\Gamma^{\prime}\right)$.

- Consider $k \in \operatorname{Char}(\Gamma)$ and write $k^{\prime}:=c(k) \in \operatorname{Char}\left(\Gamma^{\prime}\right)$. Then for any $x \in L(\Gamma)$ one has $\chi_{k}(x)=\chi_{k^{\prime}}\left(\pi^{*} x\right)$. In particular, one has a well-defined injection

$$
\pi^{*}:\left\{x \in L(\Gamma): \chi_{k}(x) \leq n\right\} \hookrightarrow\left\{y \in L\left(\Gamma^{\prime}\right): \chi_{k^{\prime}}(y) \leq n\right\} .
$$

- For any $z \in L\left(\Gamma^{\prime}\right)$ write $z$ in the form $\pi^{*} \pi_{*} z+a b_{\text {new }}$ for some $a \in \mathbb{Z}$. Then $\chi_{k^{\prime}}(z)=\chi_{k^{\prime}}\left(\pi^{*} \pi_{*} z\right)+\chi_{k^{\prime}}\left(a b_{\text {new }}\right)$. On the other hand, for any $a^{\prime} \in \mathbb{Z}$ (with $\left.\left|a^{\prime}\right| \leq|a|\right)$, one has $\chi_{k^{\prime}}\left(a^{\prime} b_{\text {new }}\right)=a^{\prime}\left(a^{\prime}+1\right) / 2 \geq 0$. In particular, if $\chi_{k^{\prime}}(z)=m$, then $z$ and $\pi^{*} \pi_{*} z$ are in the same connected component of $\left\{y \in L\left(\Gamma^{\prime}\right): \chi_{k^{\prime}}(y) \leq m\right\}$.

All these facts together imply $R_{k}=R_{k^{\prime}}$. There is a similar verification in the case when one blows up "an intersection point" corresponding to two indices $i_{0}$ and $j_{0}$ with $B\left(b_{i_{0}}, b_{j_{0}}\right)=1$. The details are left to the reader.

Finally, we verify the compatibility of the two combinatorial objects $\mathbb{H}^{+}(\Gamma,[k])$ (see Section 2.4.5) and $\mathbb{H}\left(R_{[k]}, \chi_{[k]}\right)$ (see Section 3.5). Recall that $s$ denotes the number of vertices of $\Gamma$.

4.7 Proposition For any $[k] \in \operatorname{Spin}^{c}(M)$ one has

$$
\mathbb{H}^{+}(\Gamma,[k])=\mathbb{H}\left(R_{[k]}, \chi_{[k]}\right)\left[-\max _{k^{\prime} \in[k]} \frac{\left(k^{\prime}\right)^{2}+s}{4}\right] .
$$


Proof Fix an arbitrary $k_{0} \in M_{[k]}$. Then $[k]=k_{0}+2 L$, hence $\mathbb{H}^{+}(\Gamma,[k]) \subset$ $\operatorname{Hom}\left(\right.$ Char, $\left.\mathcal{T}_{0}^{+}\right)$can be identified with a set of maps $\left\{\phi_{L}: L \rightarrow \mathcal{T}_{0}^{+}\right\}$which satisfy the following: for any $l \in L$ and $b_{j}$ with $2 n=\left(k_{0}+2 l+b_{j}, b_{j}\right)$ one has $U^{n} \cdot \phi_{L}\left(l+b_{j}\right)=\phi_{L}(l)$ if $n \geq 0$, or $\phi_{L}\left(l+b_{j}\right)=U^{-n} \cdot \phi_{L}(l)$ if $n \leq 0$. Notice that $\chi_{k_{0}}\left(l+b_{j}\right)-\chi_{k_{0}}(l)=-n$; hence the above property is equivalent with $(*)$ in Section 3.5] showing the compatibility of the two sets of restrictions. Still, one has to verify two more facts.

In order to formalize the first, let us consider the natural map $\theta: L \rightarrow \mathcal{V}\left(R_{k_{0}}\right)$, where we associate with any $l \in L$ the component of $\bar{L}_{k_{0}, \leq \chi_{k_{0}}(l)}$ containing $l$. This induces a map $\operatorname{Hom}\left(\mathcal{V}\left(R_{k_{0}}\right), \mathcal{T}_{0}^{+}\right) \rightarrow \operatorname{Hom}\left(L, \mathcal{T}_{0}^{+}\right)$by composition $\phi \mapsto$ $\phi_{L}:=\phi \circ \theta$ and, by the above compatibility verifications, a map $\theta^{*}: \mathbb{H}\left(R_{k_{0}}\right) \rightarrow$ $\mathbb{H}^{+}(\Gamma,[k])$. Since (in general) $\theta$ is not onto, one has to verify that any $\phi_{L}$ has an extension to a $\phi$ with $\theta^{*}(\phi)=\phi_{L}$, and this extension is unique.

Assume that $v \in \mathcal{V}\left(R_{k_{0}}\right) \backslash \operatorname{Im} \theta$ corresponds to a component $C_{v}$ in $\bar{L}_{k_{0}, \leq n}$. Set $m_{v}:=\max \left(\chi_{k_{0}}\left(L \cap C_{v}\right)\right.$, and take some $l_{v} \in L \cap C_{v}$ with $\chi_{k_{0}}\left(l_{v}\right)=m_{v}$. Then for any $\phi \in \mathbb{H}\left(R_{k_{0}}\right)$, the value $\phi(v)$ is uniquely determined by $\phi(v)=$ $U^{n-m_{v}} \cdot \phi_{L}\left(l_{v}\right)$.

Finally, we have to fit the gradings. With the obvious notation, for any $k=$ $k_{0}+2 l$, one has

$$
\operatorname{deg}(\phi(k))=\frac{\left(k_{0}+2 l\right)^{2}+s}{4}+d_{\mathbb{H}^{+}}=-2 \chi_{k_{0}}(l)+d_{\mathbb{H}}
$$

or, equivalently,

$$
d_{\mathbb{H}^{-}}-d_{\mathbb{H}^{+}}=\frac{k_{0}^{2}+s}{4}=\max _{k^{\prime} \in[k]} \frac{\left(k^{\prime}\right)^{2}+s}{4} .
$$

\section{$5 \quad$ Distinguished characteristic elements}

There is a more subtle way to choose a special characteristic element from each fixed orbit $[k]$, a fact which will be crucial in the next discussions. The goal of the present section is its definition.

5.1 Definitions: The canonical graded root Recall that the canonical characteristic element $K \in$ Char is defined by the equations $K\left(b_{j}\right)=-e_{j}-2$ (or equivalently, by $\left.\chi_{K}\left(b_{j}\right)=1\right)$ for any $j \in \mathcal{J}$. We denote by $\left(R_{\text {can }}, \chi_{\text {can }}\right)$ the graded root associated with $K$. In order to simplify the notation, we also write $\chi_{K}=\chi$. 
Sometimes it is preferable (motivated by the symplectic geometry) to define the "canonical" spin ${ }^{c}$ structure $\sigma_{\text {can }}$ via $c_{1}\left(\sigma_{\text {can }}\right)=[-K]$, ie identifying it by the anti-canonical bundle. Our choice above is motivated by the Riemann-Roch formulas, and the connection with singularity theory. But, in fact, from the point of view of the results of the present paper, the two choices are completely equivalent. Indeed, there is a natural involution $x \mapsto-x$ on $L, L^{\prime}$ and $H$. This is compatible with the natural involution on $\operatorname{Spin}^{c}(M)$. All the formulas in this paper are stable with respect to these involutions $\left(\mathrm{eg}, \min \chi_{K}=\min \chi_{-K}\right)$ : the orbits $[K]$ and $[-K]$ share the same properties.

5.1.1 Example If $M$ is the link of a normal surface singularity, and $\widetilde{X}$ is a resolution of $(X, 0)$, then $K$ is the first Chern class of the canonical bundle of the complex structure of $\widetilde{X} . b_{j} \in L$ denotes the fundamental class of the irreducible exceptional divisors $E_{j}$, hence $x=\sum n_{j} b_{j} \in L$ can be identified with the cycle $Z=\sum n_{j} E_{j}$ supported by the exceptional divisor. The linear equations defining $K$ are the adjunction formulas for the canonical line bundle. Hence, by Riemann-Roch, $\chi(x)$ is exactly the Euler-characteristic of the sheafcohomology of $\mathcal{O}_{Z}$.

5.2 Notation We embed $L^{\prime}$ into $L_{\mathbb{Q}}$ as in Section 2.1. We set

$$
S_{\mathbb{Q}}:=\left\{x \in L_{\mathbb{Q}}:\left(x, b_{j}\right) \leq 0 \text { for every } j \in \mathcal{J}\right\}
$$

and $S=S_{\mathbb{Q}} \cap L$. Since $B$ is negative definite, if $x \in S_{\mathbb{Q}}$, then $x \geq 0$, see Theorem 6.1 part (a).

5.3 Fix a characteristic element $k$, ie fix an $l^{\prime} \in L^{\prime}$ with $k=K+2 l^{\prime}$. Then the function $\chi_{k}: L \rightarrow \mathbb{Z}$ is equivalent to the rational valued extension of $\chi=\chi_{K}$ restricted to the sub-lattice $l^{\prime}+L$ of $L_{\mathbb{Q}}$. Indeed, extend $\chi$ by the same formula $\chi: L_{\mathbb{Q}} \rightarrow \mathbb{Q}, \chi(x):=-(K+x, x / 2)$. Then, for any $x \in L$ one has

$$
\chi_{k}(x)=\chi\left(l^{\prime}+x\right)-\chi\left(l^{\prime}\right) .
$$

In fact, the equivalence class $[k]=K+2 l^{\prime}+2 L$ can be identified with this sub-lattice $l^{\prime}+L$ in $L_{\mathbb{Q}}$. We wish to choose a distinguished element of $l^{\prime}+L$ and list some of its properties.

5.4 Lemma For any fixed $[k]=K+2\left(l^{\prime}+L\right)$, the intersection $\left(l^{\prime}+L\right) \cap S_{\mathbb{Q}}$ in $L_{\mathbb{Q}}$ admits a unique minimal element $l_{[k]}^{\prime}$. (Here minimality is considered with respect to the ordering $\leq$ in $L_{\mathbb{Q}}$.) 
Proof This is similar to Artin's proof of the existence of the fundamental cycle 11, 2]. Assume that $l^{\prime}+l_{i} \in S_{\mathbb{Q}}, l_{i} \in L$ for $i=1,2$. Set $l:=\min \left\{l_{1}, l_{2}\right\}$, and write $x_{i}=l_{i}-l \geq 0$. We show that $l^{\prime}+l \in S_{\mathbb{Q}}$ as well. Consider any $j \in \mathcal{J}$. Since $\left|x_{1}\right| \cap\left|x_{2}\right|=\emptyset$, we can assume that either $b_{j} \notin\left|x_{1}\right|$ or $b_{j} \notin\left|x_{2}\right|$. If $b_{j} \notin\left|x_{1}\right|$ then $\left(l^{\prime}+l, b_{j}\right)=\left(l^{\prime}+l_{1}, b_{j}\right)-\left(x_{1}, b_{j}\right) \leq 0$.

5.5 Definition In any fixed class $[k]$ we fix the distinguished representative $k_{r}=K+2 l_{[k]}^{\prime} \in[k]$.

5.6 Remarks (a) Since $l_{[K]}^{\prime}=0$, the distinguished representative in $[K]$ is $K$.

(b) If $e_{j} \leq-2$ for any $j$ (eg, if $\Gamma$ is a minimal resolution graph of a normal surface singularity) then $K(x) \geq 0$ for any $x \geq 0$. This fails to be true for arbitrary plumbing graphs. Still, we have $K(x)-(x, x) \geq 0$ for any $x \geq 0$. This is a special case of the following general result.

5.7 Proposition For any fixed $[k] \in \operatorname{Spin}^{c}(M)$ and the representative $k_{r} \in[k]$ one has:

(a) $\left(l_{[k]}^{\prime}, b_{j}\right) \geq b_{j}^{2}+1$ for any $j \in \mathcal{J}$;

(b) $k_{r}(x) \geq x^{2}$ for any $x \geq 0, x \in L$;

(c) $\chi_{k_{r}}(-x) \geq 0$ for any $x \geq 0, x \in L$.

Proof (a) Assume that $\left(l_{[k]}^{\prime}, b_{j_{0}}\right) \leq b_{j_{0}}^{2}$ for some $j_{0} \in \mathcal{J}$. Then by a computation $l_{[k]}^{\prime}-b_{j_{0}} \in\left(l_{[k]}^{\prime}+L\right) \cap S_{\mathbb{Q}}$, a fact which contradicts the minimality of $l_{[k]}^{\prime}$, see Lemma 5.4 .

Part (b) follows similarly. First notice that by (a) $k_{r}\left(b_{j}\right)=2\left(l_{[k]}^{\prime}, b_{j}\right)-b_{j}^{2}-2 \geq$ $b_{j}^{2}$ for any $j \in \mathcal{J}$. Therefore, if there exists an effective cycle $x>0$ with $k_{r}(x)<x^{2}$, then there exists a minimal one with this property. This means that that minimal $x>0$ satisfies

$$
x^{2}-k_{r}\left(b_{j}\right)>k_{r}(x)-k_{r}\left(b_{j}\right) \geq\left(x-b_{j}\right)^{2} \quad \text { for every } b_{j} \in|x| .
$$

Hence $0 \geq\left(l_{[k]}^{\prime}-x, b_{j}\right)$ for every $b_{j} \in|x|$. On the other hand, if $b_{j} \notin|x|$, then $0 \geq\left(l_{[k]}^{\prime}, b_{j}\right) \geq\left(l_{[k]}^{\prime}-x, b_{j}\right)$. These two inequalities show that for this $x>0$ one has $l_{[k]}^{\prime}-x \in\left(l_{[k]}^{\prime}+L\right) \cap S_{\mathbb{Q}}$, which contradicts the minimality of $l_{[k]}^{\prime}$. For (c) use (b) and the identity $2 \chi_{k_{r}}(-x)=k_{r}(x)-x^{2}$. 


\section{$6 \quad$ First properties of the graded roots $\left(R_{k}, \chi_{k}\right)$}

In this section we focus on the distinguished representatives $k_{r}=K+2 l_{[k]}^{\prime}$ of the orbits $[k]$. (Then all their properties can be transformed into similar properties of arbitrary characteristic elements via Proposition 4.4.)

For any class $[k]$, we define $S_{[k]}:=\left\{x \in L:\left(x+l_{[k]}^{\prime}, b_{j}\right) \leq 0\right.$ for every $\left.j \in \mathcal{J}\right\}$. Clearly, $S_{[K]}=S$.

6.1 Theorem (a) For any $[k]$, if $x \in S_{[k]}$ then $x \geq 0$.

(b) For any class $[k]$, consider the distinguished representative $k=k_{r}=$ $K+2 l_{[k]}^{\prime}$. Let $\bar{L}_{k, \leq n}^{s}$ be the subcomplex of $\bar{L}_{k, \leq n}$ whose 0 -skeleton consists of cycles $x \in L_{k, \leq n}^{s}:=L_{k, \leq n} \cap S_{[k]}$, and the 1-cells are exactly those 1-cells of $\bar{L}_{k, \leq n}$ which have their endpoints in $L_{k, \leq n}^{s}$. Then the natural inclusion $\bar{L}_{k, \leq n}^{s} \rightarrow \bar{L}_{k, \leq n}$ induces a surjection $\pi_{0}\left(\bar{L}_{k, \leq n}^{s}\right) \rightarrow \pi_{0}\left(\bar{L}_{k, \leq n}\right)$ for any $n$.

(c) Assume $k=K$. Then the component $C_{0}$ in $\bar{L}_{K, \leq 0}$, which contains the zero cycle, contains no non-zero effective cycle. In fact, if $\mathcal{X}$ denotes the set of non-zero cycles in the component $C_{0}$, then for any $x \in \mathcal{X}$ one has $x<0$ and $\chi(x)=0$.

(d) For $k=K, \bar{L}_{K, \leq n}$ is connected whenever $n \geq 1$.

Proof (a) Notice that $\left(l_{[k]}^{\prime}+L\right) \cap S_{\mathbb{Q}}=l_{[k]}^{\prime}+S_{[k]}$, hence (a) follows from the minimality of $l_{[k]}^{\prime}$, see Lemma [5.4. A direct argument goes as follows. Assume the contrary: $x=x_{1}-x_{2}$ with $x_{1} \geq 0, x_{2}>0$ and $\left|x_{1}\right| \cap\left|x_{2}\right|=\emptyset$. If $b_{j} \in\left|x_{2}\right|$, then $\left(b_{j}, x_{1}\right) \geq 0$, hence by the definition of $S_{[k]}$ one gets $\left(l_{[k]}^{\prime}-x_{2}, b_{j}\right) \leq$ $-\left(x_{1}, b_{j}\right) \leq 0$. If $b_{j} \notin\left|x_{2}\right|$ then $\left(x_{2}, b_{j}\right) \geq 0 \geq\left(l_{[k]}^{\prime}, b_{j}\right)$. Hence $l_{[k]}^{\prime}-x_{2} \in S_{\mathbb{Q}}$, which contradicts the minimality of $l_{[k]}^{\prime}$, see Lemma 5.4

(b) Assume that $x \in L \backslash S_{[k]}$. Then for some $j \in \mathcal{J},\left(x+l_{[k]}^{\prime}, b_{j}\right) \geq 1$. This is equivalent with $\chi_{k}\left(x+b_{j}\right) \leq \chi_{k}(x)$. If $x+b_{j} \in S_{[k]}$ then we stop, otherwise we repeat the same algorithm for $x+b_{j}$ instead of $x$. In this way we construct an increasing sequence along which $\chi_{k}$ is decreasing. Since $B$ is negative definite, this procedure must stop.

(c) We prove that any $x \in \mathcal{X}$ satisfies

(i) $x<0$ and

(ii) $\chi(x)=0$. 
First notice that $b_{j} \notin L_{K, \leq 0}$ and $-b_{j} \in L_{K, \leq 0}$ if and only if $\chi\left(-b_{j}\right)=0$, or equivalently, $e_{j}=-1$.

Since $\mathcal{X}$ represents the (non-zero) cycles of the connected component $C_{0}$, it is enough to verify the following inductive step. If $x \in \mathcal{X}$ satisfies (i) and (ii), and $x^{\prime}=x \pm b_{j} \in \mathcal{X}$, then $x^{\prime}$ satisfies (i) and (ii) as well. In order to verify (i) for $x^{\prime}$, we have to verify that the situation $x^{\prime}=x+b_{j}$ with $b_{j} \notin|x|$ is not possible. Indeed, in this case $\chi\left(x^{\prime}\right)=\chi(x)+\chi\left(b_{j}\right)-\left(x, b_{j}\right)=1+\left(-x, b_{j}\right) \geq 1$ which would contradict $x^{\prime} \in L_{K, \leq 0}$. Now, using (i) and the fact $\chi\left(x^{\prime}\right) \leq 0$, Proposition 5.7 part (c) implies $\chi\left(x^{\prime}\right)=0$.

(d) Via (a) and (b), it is enough to show that any $x_{0} \geq 0, x_{0} \in L_{K, \leq n}$, can be connected to the zero cycle by a path inside of $\bar{L}_{K, \leq n}$. For this, it is enough to verify the following two facts:

(i) For any $x>0$ with $\chi(x)>0$, there is a $b_{j} \in|x|$ such that $\chi\left(x-b_{j}\right) \leq \chi(x)$.

Indeed, if this were not the case, then we would have $\left(b_{j}, x-b_{j}\right)>1$ for any $b_{j} \in|x|$. This is equivalent with $\left(b_{j}, x\right) \geq-K\left(b_{j}\right)$, which implies $K(x)+x^{2} \geq 0$, or $\chi(x) \leq 0$, a contradiction.

(ii) For any $x>0$ with $\chi(x) \leq 0$, there exists $b_{j} \in|x|$ such that $\chi\left(x-b_{j}\right) \leq 1$.

To prove this, denote $\chi(x)$ by $c \leq 0$, write $x=\sum_{j} n_{j} b_{j}$ and set $n:=\sum_{j} n_{j}$. Clearly $n \geq 2$ since $\chi\left(b_{j}\right)=1$ for any $j$. Now, assume that $\chi\left(x-b_{j}\right)>1$ for all $b_{j} \in|x|$. Then, in the identity $-\left(b_{j}, x-b_{j}\right)=\chi(x)-\chi\left(x-b_{j}\right)-1$ the right hand side is $<c-2$. This means that $-\left(b_{j}, x\right)<c-2-e_{j}=c+K\left(b_{j}\right)$ for any $b_{j} \in|x|$. This implies that $K(x)+x^{2}+c n>0$, or $c(n-2)>0$, which is a contradiction.

Then the construction of the wanted path connecting $x_{0}$ with 0 is clear: in the path any $x$ is succeeded by some $x-b_{j}$ according to (i) or (ii).

6.2 Characterization of rational singularities Recall that a normal surface singularity is called rational if its geometric genus $p_{g}=0$. It is easy to verify that any resolution of a rational singularity is automatically good (ie any resolution graph can be considered as a plumbing graph), and the link $M$ is a rational homology sphere. Artin characterized the rational singularities topologically in terms of any fixed resolution graph $\Gamma$ [1, 2. Namely,

$$
p_{g}=0 \text { if and only if } \chi(x) \geq 1 \text { for any } x>0, x \in L .
$$

A connected, negative definite plumbing graph with this property is called a rational graph. 
For example, any plumbing graph with $-e_{j} \geq \delta_{j}$ for every $j \in \mathcal{J}$ is rational. This can be verified as follows: if $-e_{j} \geq \delta_{j}$ then the Artin's fundamental cycle $x_{\min }$ (see Remark [7.5) is exactly $\sum_{j} b_{j}$, and by an easy computation one has $\chi\left(x_{\min }\right)=1$, hence the rationality follows from Artin's criterion, see Remark [7.5 and [1, 2]. On the other hand, not any rational graph satisfies $-e_{j} \geq \delta_{j}$ : consider eg the $-E_{8}$ graph.

The class of rational graphs is closed while taking subgraphs and decreasing Euler numbers $e_{j}$.

6.3 Theorem Let $\Gamma$ be a connected, negative definite plumbing graph whose plumbed three-manifold is a rational homology sphere. Then the following facts are equivalent:

(a) $\Gamma$ is rational;

$\left(\mathrm{a}^{\prime}\right) \# \chi_{\text {can }}^{-1}(0)=1$ (where $\left(R_{\text {can }}, \chi_{\text {can }}\right)$ is the canonical graded root associated with $\Gamma)$;

(b) $R_{\text {can }}=R_{0}$ (for the definition of $R_{0}$, or of any $R_{m}$, see Example 3.4(1));

(c) $R_{\text {can }}=R_{m}$ for some $m \in \mathbb{Z}$;

(d) For all characteristic elements $k \in$ Char, $R_{k}=R_{m_{k}}$ for some $m_{k} \in \mathbb{Z}$;

( $\mathrm{Hb}) \quad \mathbb{H}\left(R_{\text {can }}\right)=\mathcal{T}_{0}^{+} ;$

(Hc) $\mathbb{H}\left(R_{\text {can }}\right)=\mathcal{T}_{m}^{+}$for some $m \in \mathbb{Z}$; or equivalently, $\mathbb{H}_{\text {red }}\left(R_{\text {can }}\right)=0 ;$

(Hd) For all $k \in$ Char, $\mathbb{H}\left(R_{k}\right)=\mathcal{T}_{m_{k}}^{+}$for some $m_{k} \in \mathbb{Z}$; or equivalently, $\bigoplus_{[k]} \mathbb{H}_{\text {red }}\left(R_{[k]}\right)=0$.

Moreover, if $\Gamma$ is rational and $k=K+2 l^{\prime}$, then

$$
m_{k}=\min \chi_{k}=\min _{x \in L} \chi\left(l^{\prime}+x\right)-\chi\left(l^{\prime}\right)=\chi\left(l_{[k]}^{\prime}\right)-\chi\left(l^{\prime}\right)=\chi_{k}\left(l_{[k]}^{\prime}-l^{\prime}\right) \leq 0 .
$$

In particular, if $\Gamma$ is rational and $k_{r}=K+2 l_{[k]}^{\prime}$, then $\min \chi_{k_{r}}=0$ (see also Proposition 4.4 and Section 4.5.1).

It is instructive to compare (a') with the property $\# \chi_{\text {can }}^{-1}(n)=1$, valid for any graph $\Gamma$ and integer $n \geq 1$ (see Theorem 6.1 part (d)).

Proof $(\mathrm{a}) \Rightarrow$ (b) follows from (1) in Section 6.2 and Theorem 6.1 (since $\bar{L}_{K, \leq 0}^{s}$ consists of only one vertex, namely $x=0$, and $\bar{L}_{K, \leq n}^{s}=\emptyset$ for $n<0$ ). (b) $\Rightarrow$ $(\mathrm{c}) \Rightarrow(\mathrm{Hc})$ and $(\mathrm{b}) \Rightarrow(\mathrm{Hb}) \Rightarrow(\mathrm{Hc})$ are clear. $(\mathrm{Hc}) \Rightarrow(\mathrm{c})$ follows from Proposition 3.5.2 Next, we verify (c) $\Rightarrow$ (a') $\Rightarrow$ (a). Indeed, if $R_{\text {can }}=R_{m}$ for 
some $m$, then clearly $m \leq \chi(0)=0$ and $\chi_{\text {can }}^{-1}(0)$ has only one element. This clearly corresponds to the connected component $C_{0}$ of $\bar{L}_{K, \leq 0}$ which contains the zero cycle. Hence $L_{K, \leq 0}=C_{0}$, and by Theorem 6.1 part (c) $\chi(x)>0$ for any $x>0$.

Finally, it is clear that $(\mathrm{d}) \Rightarrow(\mathrm{Hd}) \Rightarrow(\mathrm{Hc})$. Hence it remains to show that (a) $\Rightarrow(d)$. Using Proposition 4.4 it is enough to consider the characteristic elements of the form $k=K+2 l_{[k]}^{\prime}$. Fix such a $k$.

Let $L_{k, \leq n}^{e}=L_{k, \leq n} \cap\{$ effective cycles $\}$, and define $\bar{L}_{k, \leq n}^{e}$ similarly as $\bar{L}_{k, \leq n}^{s}$ in Theorem 6.1 part (b). Then Theorem 6.1 parts (a) and (b) show that $\pi_{0}\left(\bar{L}_{k, \leq n}^{e}\right) \rightarrow \pi_{0}\left(\bar{L}_{k, \leq n}\right)$ is onto for any $n$. Hence it is enough to show that any $x \in L_{k, \leq n}^{e}$ can be connected with the zero cycle in $\bar{L}_{k, \leq n}^{e}$ (a fact which automatically shows that $n \geq 0$ whenever $L_{k, \leq n}^{e}$ is non-empty). More precisely, we will show that for any $x>0$ there exists at least one $b_{j} \in|x|$ so that $\chi_{k}\left(x-b_{j}\right) \leq \chi_{k}(x)$. Indeed, assume that this is not true for some $b_{j}$. Then this means $\left(x+l_{[k]}^{\prime}, b_{j}\right)>1+b_{j}^{2}$ for every $b_{j} \in|x|$. Since $\left(l_{[k]}^{\prime}, b_{j}\right) \leq 0$, we get $\left(K+x, b_{j}\right) \geq 0$ for every $b_{j} \in|x|$. This implies $K(x)+x^{2} \geq 0$, or $\chi(x) \leq 0$, which contradicts the fact that $\Gamma$ is rational.

This, in particular, also shows that $\chi_{k_{r}}(x) \geq 0$ for any $x \geq 0$. From the proof of Theorem 6.1 part (b) we get that $\chi_{k_{r}}(x) \geq 0$ for any $x$. In other words, $\chi\left(l_{[k]}^{\prime}+x\right) \geq \chi\left(l_{[k]}^{\prime}\right)$ for any $x \in L$. This and Section [5.3 also imply the last statement about $m_{k}$.

6.4 Characterization of weakly elliptic singularities via $\left(R_{\text {can }}, \chi_{\text {can }}\right)$ or $\mathbb{H}\left(R_{\text {can }}\right) \quad$ A normal surface singularity is called weakly elliptic if any of its resolution graphs are weakly elliptic. A resolution graph $\Gamma$ is weakly elliptic if $\min _{x>0} \chi(x)=0$ (see [6]). (This definition is valid even if the link is not a rational homology sphere, but in order to be consistent with all the above notation, we will assume this fact as well.)

6.5 Proposition Let $\Gamma$ be a connected, negative definite plumbing graph whose plumbed three-manifold is a rational homology sphere. Then the following facts are equivalent:

(a) $\Gamma$ is weakly elliptic.

(b) $R_{\text {can }}=R\left(\left\{n_{i}\right\},\left\{n_{i j}\right\}\right)$ for some index set $I$, \#I=l+1 $\geq 2$, and $n_{i}=0$ for any $i \in I$, and $n_{i j}=1$ for any $i \neq j$.

(Hb) $\quad \mathbb{H}\left(R_{\text {can }}\right)=\mathcal{T}_{0}^{+} \oplus\left(\mathcal{T}_{0}(1)\right)^{\oplus l}$ for some $l \geq 1$. 
Proof (a) $\Leftrightarrow$ (b) follows from Theorem 6.1, and (b) $\Leftrightarrow$ (Hb) from Theorem 6.1(d) and Proposition 3.5.2.

6.6 Remarks (a) The results Theorem 6.1 part (d), Theorem 6.3 and Proposition 6.5 can also be interpreted as follows: The grading $\chi_{\text {can }}$ of $R_{\text {can }}$ satisfies $\min \chi_{\mathrm{can}} \geq 0$ (or, equivalently $\min \chi_{\mathrm{can}}=0$ ) if and only if $\Gamma$ is either rational or weakly elliptic. In this situation, if $\mathbb{H}_{\text {red }}\left(R_{\text {can }}\right)=0$ then $\Gamma$ is rational, otherwise it is weakly elliptic.

(b) Let $k_{r}=K+2 l_{[k]}^{\prime}$ as above. Then $\min \chi_{k_{r}} \geq \min \chi$. Indeed, by Theorem 6.1(a)-(b), min $\chi_{k_{r}}=\chi_{k_{r}}(x)$ for some effective cycle $x \geq 0$. But for such a cycle $\chi_{k_{r}}(x)=\chi(x)-\left(l_{[k]}^{\prime}, x\right) \geq \chi(x)$.

(c) In particular, if $\Gamma$ is rational or weakly elliptic, then for any $\operatorname{spin}^{c}$-structure $[k]$ one has $\min \chi_{k_{r}}=0$. Indeed, $0=\chi_{k_{r}}(0) \geq \min \chi_{k_{r}} \geq \min \chi=0$.

6.7 Remark One has the following connections with singularity theory. (We omit the details, since these facts are not exactly in the spirit of the present paper.) One can verify that $l$ in Proposition 6.5 is exactly the length of the elliptic sequence in the sense of S.S.-T. Yau, or, equivalently, it is the length of Laufer's sequence. (For their definitions and equivalence, see [23.) If the graph $\Gamma$ is minimally elliptic (in the sense of Laufer [6]) then $l=1$. The opposite statement is not necessarily true: one can find elliptic graphs with $l=1$ which are not numerically Gorenstein (ie $K$ is not an integral cycle), hence which are not minimally elliptic. For example, the following graph $\Gamma$ has these properties:
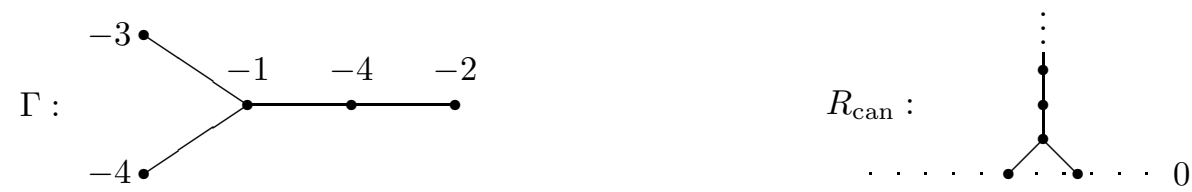

(Here the two minimal points of $R_{\text {can }}$ correspond to the zero cycle and to Artin's fundamental cycle.)

\section{Generalities about computation sequences}

7.1 The computation of the groups $\mathbb{H}$ (see Section 9) is based on the techniques of computation sequences (see, for example, [5, 6, 9, 23]). These objects were successfully used in the study of the (resolution of) normal surface singularities (see [10] for more details). Some of the next statements and proofs 
rhyme perfectly with some of those computations, eg with the proof of the existence of Artin's fundamental cycle, with Laufer's algorithm which provides this fundamental cycle, or with the construction of Yau's elliptic sequence. Nevertheless, in order to be self-contained, and since we also wish to treat the case of an arbitrary $\operatorname{spin}^{c}$-structure, we will provide all the details.

7.2 Definition Sequences $x_{0}, x_{1}, \ldots, x_{t} \in L$ with $x_{l+1}=x_{l}+b_{j(l)}(0 \leq l<t)$, where $j(l)$ is determined by some principles fixed in each individual case, are called computation sequences connecting $x_{0}$ and $x_{t}$.

In this section we will fix a $\operatorname{spin}^{c}$-structure $[k]$ and we will fix its distinguished representative $k_{r}=K+2 l_{[k]}^{\prime}$. In order to simplify the notation, in this section we write $k$ for $k_{r}$. Recall that $S_{[k]}=\left\{x \in L:\left(x+l_{[k]}^{\prime}, b_{j}\right) \leq 0\right.$ for every $\left.j \in \mathcal{J}\right\}$. We will write $m_{j}(z)$ for the coefficients of a cycle $z=\sum m_{j}(z) b_{j}$.

7.3 Lemma For any $x \in L$, there exists a unique minimal $y=y(x) \in L$ with the properties $x \leq y$ and $y \in S_{[k]}$.

Proof First notice that (since $B$ is negative definite) there exists a cycle $y>0$ so that $\left(y, b_{j}\right)<0$ for every $j \in \mathcal{J}$. Then all the coefficients of $y$ are strict positive (see also Theorem 6.1(a)). Hence, some integral multiple ay of it will satisfy $a y>x$ and $a y \in S_{[k]}$. For the existence of a unique minimal element with these properties it is enough to verify that if $y_{1}, y_{2} \in S_{[k]}$ then $y:=$ $\min \left\{y_{1}, y_{2}\right\}$ is also in $S_{[k]}$. Indeed, for any $j$, at least for one index $i \in\{1,2\}$ one has $b_{j} \notin\left|y_{i}-y\right|$. Then $\left(y+l_{[k]}^{\prime}, b_{j}\right)=\left(y_{i}+l_{[k]}^{\prime}, b_{j}\right)-\left(y_{i}-y, b_{j}\right) \leq 0$.

7.4 Lemma (Generalized Laufer's algorithm [5]) Fix an $x \in L$. Construct a computation sequence $x_{0}, \ldots, x_{t}$ by the following algorithm. Set $x_{0}=x$. Assume that $x_{l}$ is already constructed. If $x_{l} \notin S_{[k]}$, ie $\left(x_{l}+l_{[k]}^{\prime}, b_{j}\right)>0$ for some index $j$, then choose one of them for $j(l)$, and write $x_{l+1}=x_{l}+b_{j(l)}$. If $x_{l} \in S_{[k]}$, then stop and write $t=l$. Then $x_{t}$ is exactly $y(x)$ considered in Lemma 7.3

Moreover, this computation sequence satisfies $\chi_{k}\left(x_{l+1}\right) \leq \chi_{k}\left(x_{l}\right)$ for every $0 \leq$ $l<t$.

Proof We will show by induction that $x_{l} \leq y(x)$ for any $0 \leq l<t$. Indeed, assume that $x_{l} \leq y(x)$, and $x_{l+1}=x_{l}+b_{j}$. Then we have to verify that $m_{j}\left(x_{l}\right)<m_{j}(y(x))$. Assume that this is not true, ie $b_{j} \notin\left|y(x)-x_{l}\right|$. Then $\left(x_{l}+l_{[k]}^{\prime}, b_{j}\right)=\left(y(x)+l_{[k]}^{\prime}, b_{j}\right)-\left(y(x)-x_{l}, b_{j}\right) \leq 0$, a contradiction. 
On the other hand, if each $x_{l} \leq y(x)$, then the algorithm should stop with some $x_{t} \in S_{[k]}$, and $x_{t} \leq y(x)$. Then the minimality of $y(x)$ guarantees that $x_{t}=y(x)$.

The inequality follows from $\chi_{k}\left(x_{l+1}\right)-\chi_{k}\left(x_{l}\right)=1-\left(x_{l}+l_{[k]}^{\prime}, b_{j}\right) \leq 0$.

7.5 Remark (a) If we start with $x=b_{j}$ for some $j$, and $k=K$, then $y(x)$ is exactly Artin's fundamental cycle $x_{\min }$. It is the minimal strict effective cycle with $\left(x_{\min }, b_{j}\right) \leq 0$ for every $j$. Since $x_{\min } \geq \sum_{j} b_{j}$, hence also $x_{\min } \geq b_{j}$ for every $j \in \mathcal{J}$, the proof of Lemma 7.4 also shows that starting with $x_{0}=b_{j}$ we get $x_{\text {min }}$ independently of the choice of the index $j \in \mathcal{J}$. In fact, there is a computation sequence connecting $x_{0}=b_{j}$ and $x_{\text {min }}$ such that $x_{s-1}=\sum_{j} b_{j}$ and $\chi\left(x_{l}\right)=1$ for any $0 \leq j<s$, where $s=\# \mathcal{J}$.

It is known that $\Gamma$ is rational if and only if $\chi\left(x_{\min }\right)=1$ (see [1, 2]), and $\Gamma$ is weakly elliptic if and only if $\chi\left(x_{\min }\right)=0$ (see [6]). In general, $\chi\left(x_{\min }\right) \leq 1$.

(b) Assume that above $\Gamma$ is a rational graph, and $k=K$ (hence $l_{[k]}^{\prime}=0$ and $\left.\chi_{k}=\chi\right)$. Consider the computation sequence from Lemma 7.4 connecting $x_{0}:=b_{j}$ and $x_{\min }$. It satisfies $\chi\left(x_{l+1}\right) \leq \chi\left(x_{l}\right)$ for any $l$. On the other hand $\chi\left(b_{j}\right)=1$ and $\chi\left(x_{\min }\right)=1$, by the rationality of $\Gamma$. Hence $\chi\left(x_{l+1}\right)=\chi\left(x_{l}\right)$ for any $l$; in other words, $\left(x_{l}, b_{j(l)}\right)=1$ for any $l$ (a fact first noticed by Laufer [5]).

In the next paragraphs we wish to generalize the above lemmas 7.3 and 7.4 We prefer to write $\mathcal{J}=\{0\} \cup \mathcal{J}^{*}$, and hence to distinguish a base element $b_{0}$.

7.6 Lemma For any integer $i \geq 0$, there exists a unique cycle $x(i) \in L$ with the following properties:

(a) $m_{0}(x(i))=i$;

(b) $\left(x(i)+l_{[k]}^{\prime}, b_{j}\right) \leq 0$ for any $j \in \mathcal{J}^{*}$;

(c) $x(i)$ is minimal with properties (a)-(b).

Moreover, the cycle $x(i)$ satisfies $x(i) \geq 0$.

Proof First we verify that there exists at least one $x \in L$ satisfying (a) and (b). By Lemma 7.3 there exists $\widetilde{x} \in S_{[k]}$ with $\widetilde{x} \geq b_{0}$. Take an integer $a \geq 1$ such that $(a-1) l_{[k]}^{\prime}$ is an integral cycle and $h:=m_{0}\left(a \widetilde{x}+(a-1) l_{[k]}^{\prime}\right)-i \geq 0$. Then define $x:=a \widetilde{x}+(a-1) l_{[k]}^{\prime}-h b_{0}$. By a computation $m_{0}(x)=i$ and $\left(x+l_{[k]}^{\prime}, b_{j}\right)=a\left(\widetilde{x}+l_{[k]}^{\prime}, b_{j}\right)-h\left(b_{0}, b_{j}\right) \leq 0$ for $j \neq 0$. 
Next, one can verify that there exists a unique minimal element with properties (a) and (b). The proof is similar to the proof of Lemma [7.3, and it is left to the reader.

Finally we verify that $x(i) \geq 0$. Write $x(i)$ in the form $x_{1}-x_{2}$ with $x_{1} \geq 0$, $x_{2} \geq 0,\left|x_{1}\right| \cap\left|x_{2}\right|=\emptyset$. Consider an index $j \in \mathcal{J}^{*}$. If $b_{j} \notin\left|x_{1}\right|$ then one has $\left(l_{[k]}^{\prime}-x_{2}, b_{j}\right) \leq\left(l_{[k]}^{\prime}-x_{2}+x_{1}, b_{j}\right)=\left(x(i)+l_{[k]}^{\prime}, b_{j}\right) \leq 0$. Similarly, for any $b_{j} \in\left|x_{1}\right|$ one has $\left(l_{[k]}^{\prime}-x_{2}, b_{j}\right) \leq\left(l_{[k]}^{\prime}, b_{j}\right) \leq 0$. Hence $l_{[k]}^{\prime}-x_{2} \in\left(l_{[k]}^{\prime}+L\right) \cap S_{\mathbb{Q}}$, which implies $x_{2}=0$ via the minimality of $l_{[k]}^{\prime}$, see Lemma [5.4

7.7 Lemma (The computation sequence connecting $x(i)$ and $x(i+1)$ ) For any integer $i \geq 0$ consider a computation sequence constructed as follows. Set $x_{0}=x(i), x_{1}=x(i)+b_{0}$. Assume that $x_{l}(l \geq 1)$ is already constructed. If $x_{l}$ does not satisfy property $(b)$ in Lemma 7.6. then there exists some $j \in \mathcal{J}^{*}$ with $\left(x_{l}+l_{[k]}^{\prime}, b_{j}\right)>0$. Then choose one of these indices for $j(l)$, and write $x_{l+1}=x_{l}+b_{j(l)}$. If $x_{l}$ satisfies property (b) in Lemma 7.6, then stop and write $t=l$. Then $x_{t}$ is exactly $x(i+1)$.

Moreover, this computation sequence satisfies $\chi_{k}\left(x_{l+1}\right) \leq \chi_{k}\left(x_{l}\right)$ for any $0<$ $l<t$.

Corresponding to $l=0$ one has $\chi_{k}\left(x(i)+b_{0}\right)-\chi_{k}(x(i))=1-\left(x(i)+l_{[k]}^{\prime}, b_{0}\right)$. In other words, $\chi_{k}\left(x(i)+b_{0}\right)>\chi_{k}(x(i))$ if and only if $x(i) \in S_{[k]}$.

Proof Repeat the arguments used in the proof of Lemma 7.4

Lemma [7.7 has the following easy generalization (with the same proof):

7.8 Lemma Assume that $x \in L$ satisfies $m_{0}(x)=i$ and $x \leq x(i)$ for some $i \geq 0$. Consider a similar computation sequence as in Lemma 7.7 Namely, set $x_{0}=x$. Assume that $x_{l}$ is already constructed. If for some $j \in \mathcal{J}^{*}$ one has $\left(x_{l}+l_{[k]}^{\prime}, b_{j}\right)>0$ then take $x_{l+1}=x_{l}+b_{j(l)}$, where $j(l)$ is such an index $j$. If $x_{l}$ satisfies property (b) of Lemma 7.6. then stop and write $t=l$. Then $x_{t}$ is exactly $x(i)$. Moreover, this computation sequence satisfies $\chi_{k}\left(x_{l+1}\right) \leq \chi_{k}\left(x_{l}\right)$ for any $0 \leq l<t$.

Notice that, even if it is not explicitly emphasized in its notation, the cycles $\{x(i)\}_{i \geq 0}$ depend on the choice of the distinguished vertex and of the $\operatorname{spin}^{c}-$ structure $[k]$. 


\section{$8 \quad$ Almost-rational graphs, $H^{+}(-M,[k])=\mathbb{H}^{+}(\Gamma,[k])$}

8.1 Definition Assume that the plumbing graph $\Gamma$ is a negative definite connected tree. We say that $\Gamma$ is almost-rational (in short $A R$ ) if there exists a vertex $j_{0} \in \mathcal{J}$ of $\Gamma$ such that replacing $e_{j_{0}}$ by some $e_{j_{0}}^{\prime} \leq e_{j_{0}}$ we get a rational graph $\Gamma^{\prime}$. In general, the choice of $j_{0}$ is not unique. Once the distinguished vertex $j_{0}$ is fixed, we write $\mathcal{J}=\{0\} \cup \mathcal{J}^{*}$ such that the index $0 \in \mathcal{J}$ corresponds to this vertex.

8.2 Examples The set of $A R$ graphs is (surprisingly) large.

(1) All rational graphs are $A R$. Indeed, take $e_{j_{0}}^{\prime}=e_{j_{0}}$ for any vertex $j_{0}$.

(2) Any weakly elliptic graph is $A R$. This can be proved as follows. Take a computation sequence $x_{0}, \ldots, x_{t}$ for $x_{\text {min }}$ as in Remark 7.5. Since $\Gamma$ is weakly elliptic if and only if $\chi\left(x_{\min }\right)=0$ (see Remark [7.5, for example), we get from a similar discussion like in Remark 7.5 that $\left(x_{l}, b_{j(l)}\right)=1$ all the time in the computation sequence, excepting exactly one $l=l_{0}$ when this intersection $=2$ (and $b_{j\left(l_{0}\right)} \in\left|x_{l_{0}}\right|$ as well). Then take $j\left(l_{0}\right)$ for the distinguished vertex. If we replace $e_{j\left(l_{0}\right)}$ by a strict smaller integer, then for some $t^{\prime} \leq t$, the sequence $x_{0}, \ldots, x_{t^{\prime}}$ will be a computation sequence for the new fundamental cycle with $\left(x_{l}, b_{j(l)}\right)=1$ for any $l<t^{\prime}$. Hence $\chi$ of the new fundamental cycle will be 1 , a fact which characterizes the rational singularities.

(3) Any star-shaped graph is $A R$. Indeed, first blow down all the $(-1)$-vertices different from the central vertex (this transformation preserves the $A R$ graphs); let this new graph be $\bar{\Gamma}$. Then take for $j_{0}$ the central vertex of $\bar{\Gamma}$, and take for $-e_{j_{0}}^{\prime}$ an integer larger than the number of adjacent vertices of the central vertex of $\bar{\Gamma}$. Then the modified graph will become rational. This follows from the fact that a graph is rational provided that $-e_{j} \geq \delta_{j}$ is satisfied for all its vertices $j$ (see Section 6.2, or 10]). (In other words, if one takes for the distinguished vertex of $\Gamma$ the central vertex, and one takes $-e_{j_{0}}$ sufficiently large, than one gets a rational graph.)

The same argument shows that the graphs considered by Ozsváth and Szabó in 21] (and in Theorem 2.4.6) are AR.

(4) The class of $A R$ graphs is closed while taking subgraphs and decreasing the Euler numbers $e_{j}$ (since the class of rational graphs is so).

(5) Not every graph is $A R$. For example, if $\Gamma$ has two (or more) vertices, both

with decoration $-e_{j} \leq \delta_{j}-2$, then $\Gamma$ is not $A R$. For example: 
(a)

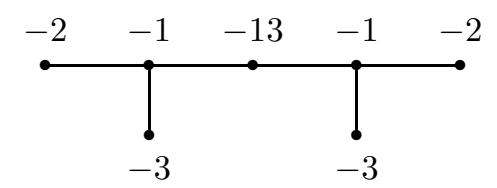

(b)

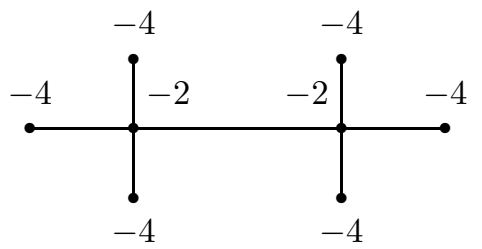

Example (a) was already considered in [21, Section 4] (see also Remark 8.4(b)). Example (b) has the following property: if we delete one of the two $(-2)-$ vertices, then all the components of the remaining graph are rational. Still, the graph itself is not $A R$.

8.3 Theorem Assume that the plumbing graph $\Gamma$ is $A R$, and let $M=M(\Gamma)$ be the oriented plumbed 3-manifold associated with it. Then for any $[k] \in$ $\operatorname{Spin}^{c}(M), H F_{\text {odd }}^{+}(-M,[k])=0$, and one has an isomorphism:

$$
H F_{\text {even }}^{+}(-M,[k])=\mathbb{H}^{+}(\Gamma,[k]) .
$$

In particular,

$$
d(M,[k])=\max _{k^{\prime} \in[k]} \frac{\left(k^{\prime}\right)^{2}+s}{4}=\frac{k_{r}^{2}+s}{4}-2 \min \chi_{k_{r}}=\frac{K^{2}+s}{4}-2 \min _{x \in L} \chi\left(l_{[k]}^{\prime}+x\right) ;
$$

and

$$
\chi\left(H F^{+}(-M,[k])\right)=\operatorname{rank}_{\mathbb{Z}} \mathbb{H}_{\text {red }}^{+}(\Gamma,[k])=\operatorname{rank}_{\mathbb{Z}} \mathbb{H}_{\text {red }}\left(R_{[k]}, \chi_{[k]}\right) .
$$

If $\Gamma$ is rational or weakly elliptic then

$$
d(M,[k])=\frac{k_{r}^{2}+s}{4}=\frac{K^{2}+s}{4}-2 \chi\left(l_{[k]}^{\prime}\right) .
$$

Proof The proof is similar to the proof of 21, Theorem 2.1] (see Theorem 2.4.6 here), but the starting point of the inductive procedure is the characterization of rational singularities in Theorem 6.3.

For any graph $\Gamma$ and vertex $j \in \mathcal{J}$, we consider the following two modified graphs:

- $\Gamma \backslash j$ is obtained from $\Gamma$ by deleting the vertex $j$ and all the edges adjacent to $j$.

- $\Gamma_{j}^{-}$is obtained from $\Gamma$ by modifying the decoration $e_{j}$ into $e_{j}-1$.

Notice that, in general, $\Gamma \backslash j$ is not connected, write $\left\{(\Gamma \backslash j)_{c}\right\}_{c}$ for its connected components. In this case, $M(\Gamma \backslash j)=\#_{c} M\left((\Gamma \backslash j)_{c}\right)$, and all the invariants $\mathrm{HF}^{+}$and $\mathbb{H}^{+}$are defined in this case as well. Moreover, one can verify that if 
$\Gamma$ is negative definite, then $\Gamma_{j}^{-}$and all the components of $\Gamma \backslash j$ are also negative definite.

The proof is based on the following commutative diagram (see [21, Section 2]). We will write $H F_{\mathrm{o} / \mathrm{e}}^{+}$for $H F_{\text {odd/even }}^{+}$.

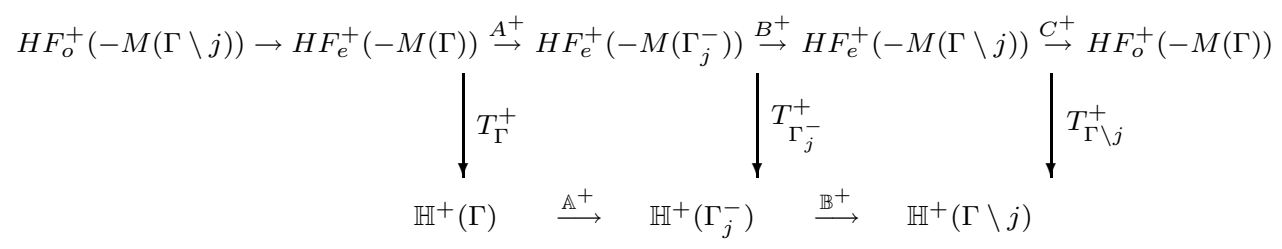

One has the following properties:

(i) the first line is exact (see [21, Section 2] and [18, Section 10.12]);

(ii) $\mathbb{B}^{+} \circ \mathbb{A}^{+}=0$, and $\mathbb{A}^{+}$is injective (see [21, Section 2.10]).

Step 1 First, let us prove that for any rational graph $\Gamma$ one has:

(a) the morphism $T_{\Gamma}^{+}: H F_{e}^{+}(-M(\Gamma)) \rightarrow \mathbb{H}^{+}(\Gamma)$ is an isomorphism,

(b) $H F_{o}^{+}(-M)=0$, in particular, $H F_{\text {red }}^{+}(-M)=0$ by Proposition 4.7 and Theorem 6.3.

If $s$ denotes the number of vertices of $\Gamma$, then this property is true for $s \leq 3$. Indeed, these cases are covered by [21] (see also Theorem 2.4.6). By induction, assume that (a) and (b) are true for any rational graph with number of vertices $<s$. Then, by the general theory (see [17, Section 7.2]), it is true even for those non-connected graphs whose connected components are rational with total number of vertices $<s$.

Consider now an arbitrary rational graph $\Gamma$ with $s$ vertices. It is well-known that if $G$ is a rational graph and $j$ is one of its vertices, , then $G_{j}^{-}$is also rational. Applying this fact several times starting from $\Gamma$, we get a graph which has the property $-e_{j} \geq \delta_{j}$ for all of its vertices. For such a graph we already know that (a) and (b) are true (see Theorem 2.4.6). Hence, going back along this sequence of graphs, we are in the following situation at each inductive step:

(iii) $T_{\Gamma_{j}^{-}}^{+}$and $T_{\Gamma \backslash j}^{+}$are isomorphisms;

(iv) $H F_{o}^{+}\left(-M\left(\Gamma_{j}^{-}\right)\right)=H F_{o}^{+}(-M(\Gamma \backslash j))=0$.

Then the following fact follows easily from the above diagram and (i)-(iii): 
(v) $\operatorname{ker} \mathbb{B}^{+}=\operatorname{Im} \mathbb{A}^{+}$.

Moreover,

(vi) $B^{+}$is onto.

This follows by a similar argument as the proof of [21, Proposition 2.8]: Since both $\Gamma$ and $\Gamma_{j}^{-}$are negative definite, the two cobordisms inducing $A^{+}$and $B^{+}$ are both negative definite, hence the third cobordism (inducing $C^{+}$) is not. In particular, it induces the trivial map on $H F^{\infty}$. But $H F_{\text {red }}^{+}(-M(\Gamma \backslash j))=0$ (by induction, since $\Gamma \backslash j$ has $<s$ vertices), it follows that $C^{+}=0$.

This induction ends the proof of step 1.

Step 2 Assume that $\Gamma$ is an $A R$ graph with distinguished vertex $j_{0}$. Then all the components of $\Gamma \backslash j_{0}$ are rational, hence $T_{\Gamma \backslash j_{0}}^{+}$is an isomorphism. Moreover, by the same argument as in (vi), we get that $C^{+}=0$ again. Then one can use a similar inductive procedure as above connecting the graph $\Gamma$ to a rational graph by a sequence of $A R$ graphs, at each time decreasing $e_{j_{0}}$ by one.

For the last formulas, see Proposition 4.7. Equation 4.5.1(4), Section 5.3 and Remark 6.6)(c).

8.4 Remarks The above proof can be pushed further to obtain the following:

(a) By the same inductive proof, one can obtain the same result as in Theorem 8.3 for a slightly larger class than $A R$ graphs. Namely, assume that $\Gamma$ has the following property: there exists a vertex $j_{0}$ such that (i) all the components of $\Gamma \backslash j_{0}$ are rational; and (ii) replacing $e_{j_{0}}$ by some $e_{j_{0}}^{\prime} \leq e_{j_{0}}$ we get an $A R$ graph. Then the statements of Theorem 8.3 are valid. Notice that the graph 8.2(5)(b) satisfies the above requirement (but it is not $A R$ ). (Nevertheless, see also Remark 9.4(c).)

(b) Assume that $\Gamma$ has the following property: there exists a vertex $j_{0}$ such that replacing $e_{j_{0}}$ by some $e_{j_{0}}^{\prime} \leq e_{j_{0}}$ we get an $A R$ graph. Then $\Gamma \backslash j_{0}$ is an $A R$ graph, and the proof of Theorem 8.3 is still valid excepting (in step 2) the triviality of $C^{+}$. Hence, for such graphs we get an isomorphism $T^{+}: H F_{\text {even }}^{+}(-M,[k]) \rightarrow \mathbb{H}^{+}(\Gamma,[k])$, but the vanishing of $H F_{\text {odd }}^{+}(-M,[k])$ does not follow. (This result and argument is the perfect analog of [21, Theorem 2.2].)

Notice that the graph $8.2(5)$ (a) satisfies the above requirement. Its homology $\mathrm{HF}^{+}$is computed in [21, Remark 4.3], and $\mathrm{HF}_{\text {odd }}^{+}$is non-zero indeed. 


\section{$9 \quad \boldsymbol{A} \boldsymbol{R}$ graphs and the computation of $\mathbb{H}\left(R_{k_{r}}, \chi_{k_{r}}\right)$}

In this section we assume that $\Gamma$ is an $A R$ graph with a distinguished vertex $0 \in \mathcal{J}$. We will use the notation $\mathcal{J}^{*}=\mathcal{J} \backslash\{0\}$. [k] will denote a fixed $\operatorname{spin}^{c}{ }_{-}$ structure with its distinguished characteristic element $k_{r}=K+2 l_{[k]}^{\prime}$. Similarly as in Section 8, in order to simplify the notation, sometimes we write $k$ for $k_{r}$. For each $i \geq 0$, we consider the cycles $x(i)$, defined in Lemma 7.6, associated with the distinguished vertex $0 \in \mathcal{J}$ and $\operatorname{spin}^{c}$-structure $[k]$.

9.1 Lemma Assume that $\Gamma$ is a $A R$ graph and fix $i \geq 0$. Then the following facts hold.

(a) Consider an arbitrary $x \in L$ with $m_{0}(x)=i$. Then $\chi_{k}(x) \geq \chi_{k}(x(i))$.

(b) Consider any $y>x(i)$ with $m_{0}(y)=m_{0}(x(i))=i$. Then there exists at least one $b_{j} \in|y-x(i)|$, for $j \in \mathcal{J}^{*}$, such that $\chi_{k}\left(y-b_{j}\right) \leq \chi_{k}(y)$.

(c) Consider a computation sequence which connects $x_{0}=x(i)$ and $x_{t}=x(i+$ $1)$, see Lemma 7.7. Then $\left(x_{l}+l_{[k]}^{\prime}, b_{j(l)}\right)=1$, or equivalently, $\chi_{k}\left(x_{l+1}\right)=\chi\left(x_{l}\right)$ for any $0<l<t$. In particular, $\chi_{k}\left(x(i)+b_{0}\right)=\chi_{k}(x(i+1))$.

Proof (a) Write $x=x(i)-y_{1}+y_{2}$ with $y_{r} \geq 0, b_{0} \notin\left|y_{r}\right|$ for $r=1,2$, and $(*)$ $\left|y_{1}\right| \cap\left|y_{2}\right|=\emptyset$. Then $\chi_{k}(x)=\chi_{k}\left(x(i)-y_{1}\right)+\chi\left(y_{2}\right)+\left(y_{1}, y_{2}\right)-\left(x(i)+l_{[k]}^{\prime}, y_{2}\right) \geq$ $\chi_{k}\left(x(i)-y_{1}\right)$. Indeed, $\left(y_{1}, y_{2}\right) \geq 0$ because of $(*)$, and $-\left(x(i)+l_{[k]}^{\prime}, y_{2}\right) \geq 0$ from the definition of $x(i)$. Finally, $\chi\left(y_{2}\right) \geq 0$, since the subgraph supported by $\left|y_{2}\right|$ can be consider as the subgraph of the modified graph $\Gamma^{\prime}$, which is rational, hence the subgraph supported by $\left|y_{2}\right|$ itself is rational. On the other hand, by Lemma 7.8 $\chi\left(x(i)-y_{1}\right) \geq \chi(x(i))$.

(b) Assume that for any $b_{j} \in|y-x(i)|$ one has $\chi_{k}\left(y-b_{j}\right)>\chi_{k}(y)$. This is equivalent with $\left(b_{j}, y+l_{[k]}^{\prime}\right) \geq 2+b_{j}^{2}$. Since $\left(b_{j}, x(i)+l_{[k]}^{\prime}\right) \leq 0$, we get that $\left(b_{j}, y-x(i)\right) \geq 2+b_{j}^{2}$, or $\left(b_{j}, y-x(i)+K\right) \geq 0$. Hence $(*) \quad \chi(y-x(i)) \leq 0$. By similar argument as in (a), $y-x(i)$ is supported by a rational graph, which contradicts $(*)$.

(c) For any $0 \leq l \leq t$ define $z_{l}:=x_{l}-x(i)$. Then clearly $z_{0}=0, z_{1}=b_{j(0)}=$ $b_{0}$, and $z_{t}=x(i+1)-x(i)$. Consider $\left(z_{l}, b_{j(l)}\right)=\left(x_{l}+l_{[k]}^{\prime}, b_{j(l)}\right)-\left(x(i)+l_{[k]}^{\prime}, b_{j(l)}\right)$. Since $\left(x_{l}+l_{[k]}^{\prime}, b_{j(l)}\right)>0$ from the construction of $\left\{x_{l}\right\},-\left(x(i)+l_{[k]}^{\prime}, b_{j(l)}\right) \geq 0$ from the definition of $x(i)$, we get $\left(z_{l}, b_{j(l)}\right)>0$. Notice that $j(l) \in \mathcal{J}^{*}$ (for $l>0)$ and $b_{0} \notin\left|z_{l}\right|$, hence the values $\left(z_{l}, b_{j(l)}\right)$ will stay unmodified even if we

replace our graph $\Gamma$ with the rational graph $\Gamma^{\prime}$ (ie if we decrease $e_{v_{0}}$ ). Since 
$\left(z_{l}, b_{j(l)}\right)>0$, we get that (in $\left.\Gamma^{\prime}\right)$ the sequence $\left\{x_{l}\right\}_{0<l \leq t}$ can be completed to a computation sequence connecting $b_{j(0)}$ and $x_{\min }$, associated with the canonical characteristic element $K$. But then Remark [7.5 shows that $\left(z_{l}, b_{j(l)}\right)=1$ for any $0<l<t$. Clearly, this is true in $\Gamma$ as well. Using again the above identity we get that $\left(x_{l}+l_{[k]}^{\prime}, b_{j(l)}\right)=1$ for any $0<l<t$, or equivalently $\chi_{k}\left(x_{l+1}\right)=\chi_{k}\left(x_{l}\right)$ (see the proof of Lemma 7.4).

Our goal is the identification of the graded tree $\left(R_{k}, \chi_{k}\right)$ (where $k=k_{r}$ ). First we identify the local minimum points $\mathcal{V}_{1}=\left\{v \in \mathcal{V}\left(R_{k}\right): \delta_{v}=1\right\}$ of $\chi_{k}$, see Section 3.3. Fix one $v \in \mathcal{V}_{1}$, and set $\chi_{k}(v)=n_{v}$. Let $C_{v}$ be the connected component of $\bar{L}_{k, \leq n_{v}}$ corresponding to $v$.

9.2 Lemma (a) $C_{v}$ has a unique maximal element $x_{v}$ (with respect to $\leq$ );

(b) $x_{v} \in S_{[k]}$;

(c) Set $i_{v}:=m_{0}\left(x_{v}\right)$. Then $x\left(i_{v}\right) \in C_{v}$.

Proof Since $v$ is a local minimum point, $(*) \chi_{k}(x)=n_{v}$ for any $x \in C_{v}$. Assume that $x, x+b_{i}$ and $x+b_{j}$ are in $C_{v}$ (with $i \neq j$ ). By $(*)$ one gets that $\left(x+l_{[k]}^{\prime}, b_{i}\right)=\left(x+l_{[k]}^{\prime}, b_{j}\right)=1$. Then by a computation, $\chi_{k}\left(x+b_{i}+b_{j}\right)=$ $\chi_{k}(x)-\left(b_{i}, b_{j}\right) \leq \chi_{k}(x)$. By the fact that $v$ is a local minimum, one gets $\chi_{k}\left(x+b_{i}+b_{j}\right)=n_{v}$. In particular, $x+b_{i}+b_{j} \in C_{v}$ as well. This is enough for (a). Indeed, in the presence of two maximal elements, one can connect them by a path in such a way that $\chi_{k}$ is constant along the path. Then any subsequence of type $x+b_{i}, x, x+b_{j}$ can be replaced by $x+b_{i}, x+b_{i}+b_{j}, x+b_{j}$. The repeated application of this leads to a contradiction. For (b) write $\chi_{k}\left(x_{v}+b_{j}\right)>\chi_{k}\left(x_{v}\right)$ for any $j$, which gives $\left(x_{v}+l_{[k]}^{\prime}, b_{j}\right) \leq 0$, and the minimal property of $x\left(i_{v}\right)$ gives $x\left(i_{v}\right) \leq x_{v}$. Notice also that $m_{0}\left(x\left(i_{v}\right)\right)=m_{0}\left(x_{v}\right)$. Using inductively part (b) of Lemma 9.1 we can construct a computation sequence connecting $x\left(i_{v}\right)$ with $x_{v}$ along which $\chi_{k}$ is non-decreasing. But since $v$ is a local minimum, $\chi_{k}$ along this computation sequence should be constant. Hence all the cycles of this sequence are in $C_{v}$; in particular, $x\left(i_{v}\right) \in C_{v}$.

In the next theorem we recover the graded tree $\left(R_{k}, \chi_{k}\right)$ from the $\chi_{k}$-values $\left\{\chi_{k}(x(i))\right\}_{i \geq 0}$ of the cycles $\{x(i)\}_{i \geq 0}$. (Recall that $k=k_{r}$.)

9.3 Theorem (a) There exists an integer $l$ such that $\chi_{k}(x(i+1)) \geq \chi_{k}(x(i))$ for any $i \geq l$. In particular, by taking $\tau(i):=\chi_{k}(x(i))$ for any $i \geq 0$, the graded tree $\left(R_{\tau}, \chi_{\tau}\right)$ is well-defined (see Example $3.4(3)$ ).

(b) $\left(R_{k}, \chi_{k}\right)=\left(R_{\tau}, \chi_{\tau}\right)$. 
Proof First we define a map $\psi: \mathcal{V}_{1}\left(R_{k}, \chi_{k}\right) \rightarrow\{x(i)\}_{i \geq 0}$ by $v \mapsto x\left(i_{v}\right)$, where $i_{v}$ is determined in Lemma 9.2 . We claim that $\psi$ is injective. Indeed, assume that for $v_{1} \neq v_{2}$ one has $i_{v_{1}}=i_{v_{2}}$. Part (c) of Lemma 9.2 guarantees that $\chi_{k}\left(v_{1}\right)=\chi_{k}\left(x\left(i_{v_{1}}\right)\right)=\chi_{k}\left(x\left(i_{v_{2}}\right)\right)=\chi_{k}\left(v_{2}\right)$. Let this integer be $n$. Consider in $\bar{L}_{k, \leq n}$ the components $C_{v_{r}}$ corresponding to $v_{r}, r=1,2$. By part (c) of Lemma $9.2 x\left(i_{v_{r}}\right) \in C_{v_{r}}$, a fact which contradicts with $C_{v_{1}} \cap C_{v_{2}}=\emptyset$.

Assume now that there exists a sequence of integers $i_{1}, i_{1}+1, \ldots, i_{2}$ such that $\chi_{k}\left(x\left(i_{1}\right)\right)>\chi_{k}\left(x\left(i_{1}+1\right)\right)=\cdots=\chi_{k}\left(x\left(i_{2}-1\right)\right)<\chi_{k}\left(x\left(i_{2}\right)\right)$. Set $n=\chi_{k}\left(x\left(i_{1}+\right.\right.$ $1))$. Then in $\bar{L}_{k, \leq n}$ there is a connected component $C_{v}$ which contains $x\left(i_{1}+1\right)$; let $v$ be the corresponding vertex of $R_{k}$. By part (a) of Lemma 9.1 $v$ is a local minimum point of $R_{k}$. Since $R_{k}$ has only a finitely many local minimum points, there exists $l$ such that for $i \geq l$ such a sequence $i_{1}, \ldots, i_{2}$ cannot occur. This, together with $\lim _{i \rightarrow \infty} \chi_{k}(x(i))=\infty$ proves part (a).

Notice also that if $v \in \mathcal{V}_{1}\left(R_{k}, \chi_{k}\right)$, then $\chi(v)=x\left(i_{v}\right)$ defines a local minimum vertex of $\left(R_{\tau}, \chi_{\tau}\right)$ This follows from the above discussion and from the properties of the computation sequences connecting the different $x(i)$ 's.

Let $\psi_{*}: \mathcal{V}_{1}\left(R_{k}\right) \rightarrow \mathcal{V}_{1}\left(R_{\tau}\right)$ be induced by $\psi$. In fact, the previous discussion also show that $\psi_{*}$ is a bijection compatible with the gradings. In order to finish the proof, consider two different local minimum points $u, v \in \mathcal{V}_{1}$ of $R_{k}$. We have to verify that $\chi_{k}(\sup (u, v))=\chi_{\tau}\left(\sup \left(\psi_{*}(u), \psi_{*}(v)\right)\right)$. Using the above notation, consider $i_{u}$ and $i_{v}$ and assume $i_{u}<i_{v}$. Then consider one index $i$ with $i_{u}<i<i_{v}$ such that $\chi_{k}(x(i)) \geq \chi_{k}(x(j))$ for any $i_{u} \leq j \leq i_{v}$. Then, in $R_{\tau}, n_{u v}=\chi_{k}\left(\sup \left(\psi_{*}(u), \psi_{*}(v)\right)\right)$ is exactly $\chi_{k}(x(i))$. We show that the same is true for $\chi_{k}(\sup (u, v))$ in $R_{k}$. Indeed, any path connecting $x\left(i_{u}\right)$ and $x\left(i_{v}\right)$ will have at least one element whose $b_{0}$-coefficient is exactly $i$ (since $\left.m_{0}(u)=i_{u}<i<i_{v}=m_{0}(v)\right)$. Hence by Lemma 9.1 part (a), $\chi_{k}(\sup (u, v)) \geq$ $\chi_{k}(x(i))$. But the iterated application of Lemma 7.7 and Lemma 9.1 part (c), provides a computation sequence connecting $x\left(i_{u}\right)$ and $x\left(i_{v}\right)$ along which $\chi_{k}$ is $\leq \chi_{k}(x(i))$; hence the equality follows.

9.4 Remarks (a) Theorem 9.3 shows that for almost rational graphs, any graded tree $\left(R_{k}, \chi_{k}\right)$ is completely determined by the values of $\chi_{k}$ along a very natural infinite computation sequence (depending on $k$ ) which contains the elements $\{x(i)\}_{i \geq 0}$. This sequence can be constructed by iterating Lemma 7.7 . ie by gluing the corresponding sequences which connect $x(i)$ with $x(i+1)$ for all $i \geq 0$.

In particular, all the important vertices of $R_{k}$ can be represented by some special elements in $L$ which can be arranged in an increasing linear order (with 
respect to $\leq$ ). (This property is extremely useful in the study of the weakly elliptic singularities [9], but here we realize that it is a more general phenomenon.)

(b) The set $\{x(i)\}_{i}$ sometimes is not very economical: only some of the $i$ 's carry substantial information which will survive in $\left(R_{\tau}, \chi_{\tau}\right)$. For example, for rational singularities, $\chi(x(i+1)) \geq \chi(x(i))$, hence only the information $\chi(x(0))=0$ is preserved in $R_{\tau}$.

(c) Part (c) of Lemma 9.1 cannot be extended for the class of graphs considered in Remark 8.4(a). In the case of that class, one can still identify $\left(R_{k}, \chi_{k}\right)$ with some $\left(R_{\tau}, \chi_{\tau}\right)$, but in the definition of $\tau$ it is not enough to take only the collection of cycles $x(i)$ along the infinite computation sequence, but one has to add some other special cycles as well.

(d) As we already mentioned, one of our interests is the numerical invariant

$$
-\mathbf{s w}_{M,[k]}^{O S z}=\chi\left(H F^{+}(-M,[k])\right)+\frac{d(M,[k])}{2} .
$$

In the case of $A R$ graphs, this equals

$$
\operatorname{rank}_{\mathbb{Z}} \mathbb{H}_{\text {red }}\left(R_{k_{r}}, \chi_{k_{r}}\right)-\min \chi_{k_{r}}+\frac{k_{r}^{2}+s}{8} .
$$

The term $k_{r}^{2}+s$ (from $K^{2}+s$ and $l_{[k]}^{\prime}$ ) is clear. The next fact provides the other term.

9.5 Corollary For any $\operatorname{spin}^{c}$-structure $[k]$ consider the representative $k_{r}=$ $K+2 l_{[k]}^{\prime}$. Then

$$
\operatorname{rank}_{\mathbb{Z}} \mathbb{H}_{\text {red }}\left(R_{k_{r}}, \chi_{k_{r}}\right)-\min \chi_{k_{r}}=\sum_{i \geq 0} \max \left\{0,-1+\left(b_{0}, x(i)+l_{[k]}^{\prime}\right)\right\} .
$$

(In fact, $\operatorname{rank}_{\mathbb{Z}} \mathbb{H}_{r e d}\left(R_{k^{\prime}}, \chi_{k^{\prime}}\right)$ is independent of the choice of $k^{\prime} \in[k]$.)

Proof We apply Corollary 3.7. Indeed, using Lemma 9.1 part (c),

$$
\chi_{k}(x(i))-\chi_{k}(x(i+1))=\chi_{k}(x(i))-\chi_{k}\left(x(i)+b_{0}\right)=-1+\left(b_{0}, x(i)+l_{[k]}^{\prime}\right) .
$$

9.6 Corollary Consider a normal surface singularity $(X, 0)$, and $\widetilde{X}$ be a fixed resolution of it. Consider any holomorphic line bundle $\mathcal{L} \in \operatorname{Pic}(\widetilde{X})$ on $\widetilde{X}$ with $c_{1}(\mathcal{L})=-l_{[k]}^{\prime}$ (here $L^{\prime} \approx H^{2}(\widetilde{X}, \mathbb{Z})$ by Poincaré duality). Assume that the resolution graph is $A R$. Then

(a) $h^{1}(\widetilde{X}, \mathcal{L}) \leq \operatorname{rank}_{\mathbb{Z}} \mathbb{H}_{r e d}\left(R_{k_{r}}, \chi_{k_{r}}\right)-\min \chi_{k_{r}}$. 
(b) If $(X, 0)$ is rational then both sides of this inequality (a) are zero.

(c) If $(X, 0)$ is weakly elliptic, and $[k]=[K]$, then $\operatorname{rank}_{\mathbb{Z}} \mathbb{H}_{\text {red }}\left(R_{\text {can }}\right)=l$ and $\min \chi_{\text {can }}=0$ (see Proposition 6.5); in particular $p_{g} \leq l$.

This answers positively the inequality part of the main conjecture [12, modified as in Problem 2.5 (IV) for $A R$ resolution graphs. For Gorenstein weakly elliptic singularities, the equality $p_{g}=l$ follows from [9]. For a generalization of the conjecture [12] and the construction of those line bundles $\mathcal{L}$ for which one expects equality above, see [1].

Proof We need only to prove part (a). For this use the above infinite computational sequence $\left\{x_{l}\right\}_{l}$ (see Remark 9.4(a)). First notice that (using the theorem on formal functions, and the identification of the elements $x_{l}=\sum_{j} n_{j} b_{j} \in L$ with cycles $\sum_{j} n_{j} E_{j}$, see Example 5.1.1), one has

$$
H^{1}(\widetilde{X}, \mathcal{L})=\lim _{\leftarrow} H^{1}\left(x_{l},\left.\mathcal{L}\right|_{x_{l}}\right) .
$$

Next, consider the cohomology long exact sequences associated with the short exact sequences obtained from

$$
0 \rightarrow \mathcal{O}_{b_{i(l)}}\left(-x_{l}\right) \rightarrow \mathcal{O}_{x_{l+1}} \rightarrow \mathcal{O}_{x_{l}} \rightarrow 0
$$

by tensoring with $\mathcal{L}$. This provides the exact sequence

$$
\rightarrow H^{1}\left(\mathbb{P}^{1}, \mathcal{O}_{\mathbb{P}^{1}}\left(-\left(b_{i(l)}, x_{l}+l_{[k]}^{\prime}\right)\right)\right) \rightarrow H^{1}\left(\left.\mathcal{L}\right|_{x_{l+1}}\right) \rightarrow H^{1}\left(\left.\mathcal{L}\right|_{x_{l}}\right) \rightarrow 0 .
$$

Then use Lemma 9.1 part (c) and Corollary 9.5

\section{Example: Lens spaces}

10.1 Notation We invite the reader to refresh the notation of Section 2.1 In particular, we recall that $\left\{g_{j}\right\}_{j \in \mathcal{J}}$ denotes the dual base in $L^{\prime}$, see the last paragraph of Section 2.1]

10.2 The plumbing graph of lens spaces Consider the continued fraction

$$
\frac{p}{q}=\left[k_{1}, k_{2}, \ldots, k_{s}\right]:=k_{1}-\frac{1}{k_{2}-\frac{1}{\ddots \cdot-\frac{1}{k_{s}}}},
$$

where $k_{j} \geq 2$ for any $j$. Then 


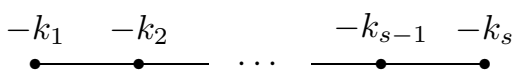

is the (minimal) plumbing graph $\Gamma$ of the lens space $M=L(p, q) . \quad M$ (and $\Gamma$ ) can be considered as the link (respectively, the minimal resolution graph) of the cyclic quotient singularity $\mathbb{C}^{2} / \mathbb{Z}_{p}$, where the action is $\xi *(x, y)=\left(\xi x, \xi^{q} y\right)$. $M$ can also be obtained as a $-p / q$ surgery on the unknot in $S^{3}$.

For any $1 \leq i \leq j \leq s$ we write the continued fraction $\left[k_{i}, \ldots, k_{j}\right]$ as a rational number $n_{i j} / d_{i j}$ with $n_{i j}>0$ and $\operatorname{gcd}\left(n_{i j}, d_{i j}\right)=1$. We also set $n_{i, i-1}:=1$ and $n_{i j}:=0$ for $j<i-1$. Since $n_{i j} / d_{i j}=k_{i}-d_{i+1, j} / n_{i+1, j}$, one gets $d_{i j}=n_{i+1, j}$ and $n_{i j}=k_{i} n_{i+1, j}-n_{i+2, j}$ for any $i \leq j$. In fact, any such identity has its symmetric version. For this it is helpful to notice that $n_{i j}$ is the determinant of $-B_{(i, j)}$, where $B_{(i, j)}$ is the bilinear form associated with the graph

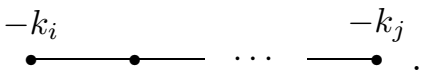

This symmetry in the present case reads as: $n_{i j}=k_{j} n_{i, j-1}-n_{i, j-2}$ for any $i \leq j$. Using these, for any $1 \leq j \leq s$ one obtains by induction:

$$
n_{1 j} \cdot n_{2 s}=p \cdot n_{2 j}+n_{j+2, s} \text { and } n_{j s} n_{1, s-1}=p \cdot n_{j, s-1}+n_{1, j-2} .
$$

Clearly $n_{1 s}=p$ and $n_{2 s}=q$. We write $q^{\prime}:=n_{1, s-1}$. Then by (1) $q q^{\prime} \equiv 1$ modulo $p$ (and $0<q^{\prime}<p$ ).

10.3 The group $H$, the $\operatorname{spin}^{c}$ structures, and the elements $l_{[k]}^{\prime}$ Clearly $L^{\prime} / L=H=\mathbb{Z}_{p}$, and $\left[g_{s}\right]=g_{s}+L$ is one of its generators (with this choice we can use the present formulas in the next section as well). Indeed, $\left[g_{j}\right]=$ $\left[n_{j+1, s} g_{s}\right]$ in $H(1 \leq j \leq s)$.

Similarly, the set of $\operatorname{spin}^{c}$-structures on $M$ is the set of orbits $\left\{\left[-a g_{s}\right]\right\}_{0 \leq a<p}=$ $\left\{-a g_{s}+L\right\}_{0 \leq a<p}$ (we prefer to use this sign, since $-g_{s}$ is effective). More precisely, this correspondence is $[k]=K+2\left(-a g_{s}+L\right)$, where $a$ runs from 0 to $p-1$. In order to emphasize the role of $a$, we also use the notation $l_{\left[-a g_{s}\right]}^{\prime}$ for $l_{[k]}^{\prime}$.

For any $0 \leq a<p$ write

$$
l_{\left[-a g_{s}\right]}^{\prime}=-\left(a_{1} g_{1}+a_{2} g_{2}+\cdots+a_{s} g_{s}\right) .
$$

Since $\left(l_{\left[-a g_{s}\right]}^{\prime}, b_{j}\right) \leq 0$, we get $a_{j} \geq 0$ for any $j$. In the next discussion we will clarify the relationship between the integer $0 \leq a<p$ (which codifies $\left.\operatorname{Spin}^{c}(L(p, q))\right)$ and the system $E(a):=\left(a_{1}, \ldots, a_{s}\right)$ (the coefficients of the corresponding minimal vectors $\left.l_{\left[-a g_{s}\right]}^{\prime}\right)$. 
10.3.1 Lemma For $i \leq j$ one has $\sum_{t=i}^{j} n_{i, t-1} a_{t}<n_{i j}$ and $\sum_{t=i}^{j} n_{t+1, j} a_{t}<$ $n_{i j}$.

Proof We verify the second set of inequalities by induction over $j-i$. If $i=j$, then consider $v:=l_{\left[-a g_{s}\right]}^{\prime}-b_{i} \in l_{\left[-a g_{s}\right]}^{\prime}+L$. Clearly $\left(v, b_{t}\right) \leq 0$ for any $t \neq i$. Since $l_{\left[-a g_{s}\right]}^{\prime}$ is minimal in $\left(l_{\left[-a g_{s}\right]}^{\prime}+L\right) \cap S_{\mathbb{Q}}$, we get that $\left(v, b_{i}\right)$ should be positive. Hence $-a_{i}+k_{i}>0$.

Next, for the case $j=i+1$, notice that by the inductive step, $l:=\left(k_{i}-a_{i}\right) b_{i+1}+$ $b_{i}>0$. Then consider $v:=l_{\left[-a g_{s}\right]}^{\prime}-l \in l_{\left[-a g_{s}\right]}^{\prime}+L$. Then for any $t \neq j$, the value $\left(v, b_{t}\right) \leq 0$ automatically. Again by the minimality of $l_{\left[-a g_{s}\right]}^{\prime}$, we get that $\left(v, b_{j}\right)>0$, or $n_{i j}-n_{j j} a_{i}-a_{j}>0$. The induction follows similarly.

In particular, the entries of $\left(a_{1}, \ldots, a_{s}\right)$ satisfy the system of inequalities:

$$
\left\{\begin{array}{l}
a_{1} \geq 0, \ldots, a_{s} \geq 0 \\
n_{i+1, s} a_{i}+n_{i+2, s} a_{i+1}+\cdots+n_{s s} a_{s-1}+a_{s}<n_{i s} \text { for any } 1 \leq i \leq s .
\end{array}\right.
$$

By this system one can identify the possible $\operatorname{spin}^{c}$-structures with the possible combinations $\left(a_{1}, \ldots, a_{s}\right)$. For this, first notice that the integer $a$ can be recovered from $\left(a_{1}, \ldots, a_{s}\right)$ by

$$
a=n_{2 s} a_{1}+n_{3 s} a_{2}+\cdots+n_{s s} a_{s-1}+a_{s} .
$$

Indeed, modulo $L$, one has the following identities:

$$
a g_{s} \equiv-l_{\left[-a g_{s}\right]}^{\prime} \equiv \sum_{j=1}^{s} a_{j} g_{j} \equiv\left(\sum_{j=1}^{s} a_{j} n_{j+1, s}\right) g_{s} .
$$

Since $\sum a_{j} n_{j+1, s}<p$ (from (SI), with $i=1$ ), the above identity follows.

Next, any $0 \leq a<p$ determines inductively the entries $a_{1}, \cdots, a_{s}$ by the formula

$$
a_{i}=\left[\frac{a-\sum_{t=1}^{i-1} n_{t+1, s} a_{t}}{n_{i+1, s}}\right] \quad(1 \leq i \leq s) .
$$

In fact, this also shows that the set of integral solutions of the system (SI) is exactly the set of all possible combinations $\left(a_{1}, \ldots, a_{s}\right)$ associated with the integers $0 \leq a<p$ by the above procedure.

10.3.2 As a curiosity, we mention that the above system (SI) can also be interpreted in language of "generalized" continued fractions. For any $1 \leq i \leq s$ 
write $r_{i}:=n_{i s} / n_{i+1, s}$. Then

$$
\frac{a}{p}=\frac{a_{1}+\frac{a_{2}+\frac{a_{s-1}+\frac{a_{s}}{r_{s}}}{\ldots}}{r_{3}}}{r_{1}} .
$$

The inequalities (SI) imply that all the possible fractions in the above expression are $<1$; and this property guarantees the uniqueness of the entries $\left(a_{1}, \ldots, a_{s}\right)$ in this continued fraction (for any given $0 \leq a<p$ ).

10.3.3 In fact,

$$
l_{\left[-(p-1) g_{s}\right]}^{\prime}=g_{s}+\sum_{i=1}^{s} b_{i}=-\left(\left(k_{1}-1\right) g_{1}+\sum_{i=2}^{s}\left(k_{i}-2\right) g_{i}\right) .
$$

This follows from the fact that $-(p-1) g_{s} \equiv g_{s}$ modulo $L$, and $g_{s}+\sum b_{j}$ is in the unit cube $\sum r_{j} b_{j}, 0<r_{j}<1$ for any $1 \leq j \leq s$. (Notice that in general it is not true that $l_{\left[-a g_{s}\right]}^{\prime}$ is in the unit cube.) (1) can be generalized by the following algorithm and identity (the verification is left to the reader).

The set of all possible combinations $E(a)=\left(a_{1}, \ldots, a_{s}\right), 0 \leq a<p$, in the order $E(p-1), \ldots, E(0)$, can be generated inductively as follows. Start with $E(p-1)=\left(k_{1}-1, k_{2}-2, \ldots, k_{s}-2\right)$. Assume that $E(a)=\left(a_{1}, \ldots, a_{s}\right)$ is already determined. Then, if $a_{s}>0$, then $E(a-1)=\left(a_{1}, \ldots, a_{s-1}, a_{s}-1\right)$. If $a_{i}=\cdots=a_{s}=0$, but $a_{i-1} \neq 0$, then

$$
E(a-1)=\left(a_{1}, \ldots, a_{i-2}, a_{i-1}-1, k_{i}-1, k_{i+1}-2, \ldots, k_{s}-2\right) .
$$

In particular

$$
l_{\left[-(a-1) g_{s}\right]}^{\prime}-l_{\left[-a g_{s}\right]}^{\prime}=g_{s}+\sum_{t=i}^{s} b_{t} .
$$

Before we start the list of invariants, we prove the following identities for $E(a)=$ $\left(a_{1}, \ldots, a_{s}\right)$. In the sequel $\{x\}:=x-[x]$ denotes the fractional part of the real number $x$.

10.4 Lemma $\left[a q^{\prime} / p\right]=\sum_{t=1}^{s} a_{t} n_{t+1, s-1}$ and $\left\{a q^{\prime} / p\right\}=\left(\sum_{t=1}^{s} a_{t} n_{1, t-1}\right) / p$.

Proof Via Equation (1) in Lemma 10.3.1 we have to show that

$$
\sum_{t=1}^{s} a_{t} n_{t+1, s-1} \leq \frac{n_{1, s-1}}{p} \sum_{t=1}^{s} a_{t} n_{t+1, s}<1+\sum_{t=1}^{s} a_{t} n_{t+1, s-1} .
$$


This follows from the second part of (1) in Section 10.2 and the first inequality of Lemma 10.3.1 (applied for $(i, j)=(1, s)$ ). The second part follows from the expression of $\left[a q^{\prime} / p\right]$ and the second part of (1) in Section [10.2.

10.5 The Ozsváth-Szabó invariant $H F^{+}( \pm M)$ Since $\Gamma$ is rational, by Theorem 6.3 and Theorem 8.3. $H F_{\text {red }}^{+}( \pm M)=0$ and $H F^{+}( \pm M,[k])=\mathcal{T}_{ \pm d}^{+}$, where

$$
d:=d(M,[k])=\frac{k_{r}^{2}+s}{4}=\frac{K^{2}+s}{4}-2 \chi\left(l_{[k]}^{\prime}\right) .
$$

Notice that (see eg [12, Section 7.1]):

$$
\frac{K^{2}+s}{4}=\frac{p-1}{2 p}-3 \cdot s(q, p),
$$

where $s(q, p)$ denotes the Dedekind sum

$$
\boldsymbol{s}(q, p)=\sum_{l=0}^{p-1}\left(\left(\frac{l}{p}\right)\right)\left(\left(\frac{q l}{p}\right)\right), \text { where }((x))=\left\{\begin{array}{cll}
\{x\}-1 / 2 & \text { if } & x \in \mathbb{R} \backslash \mathbb{Z} \\
0 & \text { if } & x \in \mathbb{Z} .
\end{array}\right.
$$

(In fact, this formula for $K^{2}+s$ for cyclic quotients goes back to the work of Hirzebruch.)

Next, we wish to determine $\chi\left(l_{\left[-a g_{s}\right]}^{\prime}\right)$.

10.5.1 Proposition For any $0 \leq a<p$ one has

$$
\chi\left(l_{\left[-a g_{s}\right]}^{\prime}\right)=\frac{a(1-p)}{2 p}+\sum_{j=1}^{a}\left\{\frac{j q^{\prime}}{p}\right\} .
$$

Proof Using the notation of Section 10.3.3 (in particular $a_{t}=0$ for $t \geq i$ ), we have

$$
\begin{gathered}
\chi\left(l_{\left[-(a-1) g_{s}\right]}^{\prime}\right)=\chi\left(l_{\left[-a g_{s}\right]}^{\prime}+g_{s}+\sum_{t=i}^{s} b_{t}\right)= \\
\chi\left(l_{\left[-a g_{s}\right]}^{\prime}\right)+\chi\left(g_{s}+\sum_{t=i}^{s} b_{t}\right)-\left(l_{\left[-a g_{s}\right]}^{\prime}, g_{s}+\sum_{t=i}^{s} b_{t}\right) .
\end{gathered}
$$

Since $l_{\left[-a g_{s}\right]}^{\prime}=-\sum_{t=1}^{i-1} a_{t} g_{t}$ and $-p g_{s}=\sum_{j} n_{1, j-1} b_{j}$, and from Lemma 10.4 one gets

$$
-\left(l_{\left[-a g_{s}\right]}^{\prime}, g_{s}+\sum_{t=i}^{s} b_{t}\right)=\sum_{t} a_{t}\left(g_{t}, g_{s}\right)=-\sum_{t} a_{t} n_{1, t-1} / p=-\left\{a q^{\prime} / p\right\} .
$$


Moreover, $\chi\left(g_{s}+\sum_{t=i}^{s} b_{t}\right)=\chi\left(g_{s}\right)+\chi\left(\sum_{t=i}^{s} b_{t}\right)-1=\chi\left(g_{s}\right)$ by an easy induction. Notice that the $b_{s}$-coefficient of $K\left(\right.$ ie $\left.\left(K, g_{s}\right)\right)$ is $-1+\left(q^{\prime}+1\right) / p$ (see [12, Section 5.2]), and $g_{s}^{2}=-q^{\prime} / p$, hence $\chi\left(g_{s}\right)=(p-1) /(2 p)$. Hence the formula follows by induction over $a\left(\right.$ since $\left.\chi\left(l_{[0]}^{\prime}\right)=0\right)$.

10.5.2 Clearly, with the choice $g_{1}$ as a generator, by a similar proof one has

$$
\chi\left(l_{\left[-a g_{1}\right]}^{\prime}\right)=\frac{a(1-p)}{2 p}+\sum_{j=1}^{a}\left\{\frac{j q}{p}\right\} .
$$

10.6 The Casson-Walker invariant is $\lambda(L(p, q))=p \cdot \boldsymbol{s}(q, p) / 2$ (see, for example, [12, Section 5.3]).

10.7 The Reidemeister-Turaev torsion One can use for the computation of the sign-refined Reidemeister-Turaev torsion the formula [12, Section 5.7] (see also [12, Section 3.5]). In that article, the canonical $\operatorname{spin}^{c}$-structure $\sigma_{\text {can }}$ was defined via $c_{1}\left(\sigma_{\text {can }}\right)=-K$, and an arbitrary $\operatorname{spin}^{c}$ structure via the action $\sigma=h_{\sigma} \cdot \sigma_{\text {can }}$ for some $h_{\sigma} \in H$. This reads as $-c_{1}(\sigma)=-c_{1}\left(\sigma_{\text {can }}\right)-2 h_{\sigma}$, which in our case corresponds to $k_{r}=K+2 l_{[k]}^{\prime}$. In particular, for $\left[-a g_{s}\right], h_{\sigma}$ is the class of $a g_{s}$ (see also Section 5.1).

Then we apply [12, Theorem 5.7]. Here we identify any character $\chi$ of $H$ by the root of unity $\xi:=\chi\left(\left[g_{s}\right]\right)$. Then we get:

$$
\mathcal{T}_{M,\left[-a g_{s}\right]}(1)=\frac{1}{p} \sum \frac{\xi^{-a}}{(\xi-1)\left(\xi^{q}-1\right)},
$$

where the sum is over all $p$-roots of unity $\xi$ with $\xi \neq 1$. If $a=0$ then

$$
\mathcal{T}_{M,[0]}(1)=\frac{1}{p} \sum \frac{1}{\left(\xi^{q}-1\right)(\xi-1)}=\frac{p-1}{4 p}-s(q, p) .
$$

For $a>0$, we use the same inductive step as above, namely

$$
\mathcal{T}_{M,\left[-(a-1) g_{s}\right]}(1)-\mathcal{T}_{M,\left[-a g_{s}\right]}(1)=\frac{1}{p} \sum \frac{\xi^{-a}}{\xi^{q}-1}=\frac{1}{p} \sum \frac{\xi^{-a q^{\prime}}}{\xi-1}=\left\{\frac{a q^{\prime}}{p}\right\}+\frac{1-p}{2 p} .
$$

Adding all these identities, and using Proposition 10.5.1 one gets

$$
\mathcal{T}_{M,\left[-a g_{s}\right]}(1)=\frac{p-1}{4 p}-\boldsymbol{s}(q, p)-\chi\left(l_{\left[-a g_{s}\right]}^{\prime}\right) .
$$

10.8 The identity $\mathbf{s w}_{M,[k]}^{O S Z}=\mathbf{s w}_{M,[k]}^{T C W}$ Using the above formulas, one gets

$$
\mathcal{T}_{M,[k]}(1)-\frac{\lambda(M)}{|H|}=\frac{d(M,[k])}{2},
$$


which proves the identity $\mathbf{s w}_{M,[k]}^{O S z}=\mathbf{s w}_{M,[k]}^{T C W}$ mentioned in Section 2.5

Since $\sum_{[k]} \mathcal{T}_{M,[k]}(1)=0$, one also gets

$$
-\lambda(M)=\sum_{[k] \in \operatorname{Spin}^{c}(M)} \frac{k_{r}^{2}+s}{8},
$$

or, equivalently,

$$
\sum_{[k]} \chi\left(l_{[k]}^{\prime}\right)=\frac{p-1}{4}-p \cdot s(q, p) .
$$

\section{Example: Seifert 3-manifolds}

11.1 In this section we will use mainly the notation of 13] (which also provides a list of classical references for Seifert 3-manifolds).

Assume that $M$ is a Seifert rational homology sphere with negative orbifold Euler number $e$ (equivalently, the corresponding plumbing graph is negative definite). The minimal plumbing graph $\Gamma$ is star-shaped with $\nu$ arms; we assume $\nu \geq 3$. (The case $\nu<3$ is completely covered by the previous section.) $\Gamma$ can be determined from the (normalized) Seifert invariants $\left\{\left(\alpha_{l}, \omega_{l}\right)\right\}_{l=1}^{\nu}$ and $e$ as follows. Here $0 \leq \omega_{l}<\alpha_{l}$ for any $1 \leq l \leq \nu$, and $e=e_{0}+\sum_{l} \omega_{l} / \alpha_{l}$, where $e_{0}$ is the decoration of the central vertex. The other decorations can be determined from the continued fraction $\alpha_{l} / \omega_{l}=\left[k_{1}^{l}, k_{2}^{l}, \cdots, k_{s_{l}}^{l}\right]$; the vertices $\left\{v_{j}^{l}\right\}_{j=1}^{s_{l}}$ situated on the $l^{t h}$-arm are decorated by $e_{j}^{l}=-k_{j}^{l}$ in such a way that the vertex $v_{1}^{l}$ is connected with the central vertex.

We denote by $b_{0}$ and $b_{j}^{l}\left(1 \leq j \leq s_{l} ; 1 \leq l \leq \nu\right)$ the base elements of $L$, respectively by $g_{0}$ and $\left\{g_{j}^{l}\right\}_{j l}$ the dual base elements of $L^{\prime}$ (see Section 2.1). As we already mentioned (see Example $8.2(3)$ ), such a graph $\Gamma$ is $A R$ with its central vertex distinguished.

We will also use the following notation: $\varepsilon:=\left(2-\nu+\sum_{l} 1 / \alpha_{l}\right) / e$ denotes the "exponent of $M$ " (in some articles $-\varepsilon$ is the "log discrepancy of the central vertex"). For any $l$ we consider $0<\omega_{l}^{\prime}<\alpha_{l}$ with $\omega_{l} \omega_{l}^{\prime} \equiv 1$ modulo $\alpha_{l}$.

The following identities are well-known (they can be deduced from [12, Section 5], for example).

$$
\left(g_{0}, g_{0}\right)=\frac{1}{e} ;\left(g_{0}, g_{s_{l}}^{l}\right)=\frac{1}{e \alpha_{l}} ;\left(g_{s_{t}}^{t}, g_{s_{l}}^{l}\right)=\frac{1}{e \alpha_{t} \alpha_{l}}(t \neq l) ;\left(g_{s_{l}}^{l}, g_{s_{l}}^{l}\right)=\frac{1}{e \alpha_{l}^{2}}-\frac{\omega_{l}^{\prime}}{\alpha_{l}} .
$$




$$
\begin{gathered}
-\left(K, g_{0}\right)=1+\varepsilon ; \quad-\left(K, g_{s_{l}}^{l}\right)=1+\frac{\varepsilon-\omega_{l}^{\prime}}{\alpha_{l}} . \\
\chi\left(g_{0}\right)=\frac{1}{2}+\frac{\varepsilon}{2}-\frac{1}{2 e} ; \quad \chi\left(g_{s_{l}}^{l}\right)=\frac{1}{2}+\frac{\varepsilon}{2 \alpha_{l}}-\frac{1}{2 e \alpha_{l}^{2}} .
\end{gathered}
$$

11.2 Notation For any fixed $1 \leq l \leq \nu$, we consider similar notation as in the previous section. For any $1 \leq i \leq j \leq s_{l}$, we write $n_{i j}^{l} / d_{i j}^{l}:=\left[k_{i}^{l}, \ldots, k_{j}^{l}\right]$ (with $n_{i j}^{l}>0$ and $\operatorname{gcd}\left(n_{i j}^{l}, d_{i j}^{l}\right)=1$ ); and we set $n_{i, i-1}^{l}:=1$ and $n_{i j}^{l}:=0$ for $j<i-1$. Clearly they satisfy the identities considered in Section 10.2 Moreover, $\alpha_{l}=n_{1, s_{l}}^{l}, \omega_{l}=n_{2, s_{l}}^{l}$, and $\omega_{l}^{\prime}=n_{1, s_{l}-1}^{l}$.

11.3 The group $H$ In the abelian group $H=L^{\prime} / L$ one has

$$
\left[g_{j}^{l}\right]=\left[n_{j+1, s_{l}}^{l} g_{s_{l}}^{l}\right] .
$$

In fact, one has the following (Abelian) presentation (see [15]):

$$
\left.H=\left\langle g_{0}, g_{s_{1}}^{1}, g_{s_{2}}^{2}, \ldots g_{s_{\nu}}^{\nu}\right| e_{0} g_{0}+\sum_{l=1}^{\nu} \omega_{l} g_{s_{l}}^{l}=0, \quad g_{0}=\alpha_{l} g_{s_{l}}^{l} \text { for all } l\right\rangle \text {. }
$$

The order of $H$ is $-e \alpha_{1} \cdots \alpha_{\nu}$, and the order $o$ of the subgroup $\left\langle g_{0}\right\rangle$ is $o=-e \alpha$, where $\alpha:=\operatorname{lcm}\left(\alpha_{1}, \ldots, \alpha_{\nu}\right)$ (see [15]).

11.4 $\operatorname{Spin}^{c}$-structures and representatives $l_{[k]}^{\prime}$ We fix a $\operatorname{spin}^{c}$-structure $[k]=K+2\left(l_{[k]}^{\prime}+L\right)$, or equivalently, the orbit $l_{[k]}^{\prime}+L$ in $L^{\prime} / L$. We assume that the distinguished representative $l_{[k]}^{\prime} \in L^{\prime}$ has the following form

$$
l_{[k]}^{\prime}=-a_{0} g_{0}-\sum_{j, l} a_{j}^{l} g_{j}^{l} .
$$

For any fixed $1 \leq l \leq \nu$, we associate with the collection $\left(a_{1}^{l}, \ldots, a_{s_{l}}^{l}\right)$ the integer (see Section 10.3):

$$
a_{l}:=\sum_{t \geq 1} n_{t+1, s_{l}}^{l} a_{t}^{l}
$$

The integers $\left\{a_{0} ; a_{j}^{l}\right\}_{l j}$, respectively $\left\{a_{0} ; a_{l}\right\}_{l}$ satisfy the following inequalities:

11.5 Proposition Consider $l_{[k]}^{\prime}$ and the notation (1) and (2) above. Then

$$
\begin{cases}a_{0} \geq 0 ;, a_{j}^{l} \geq 0 & \left(1 \leq l \leq \nu, 1 \leq j \leq s_{l}\right) ; \\ \widetilde{a}_{j}^{l}:=\sum_{t \geq j} n_{t+1, s_{l}}^{l} a_{t}^{l}<n_{j s_{l}}^{l} & \left(1 \leq l \leq \nu, 1 \leq j \leq s_{l}\right) ; \\ 1+a_{0}+i e_{0}+\sum_{l}\left[\frac{i \omega_{l}+a_{l}}{\alpha_{l}}\right] \leq 0 & \text { for any } i>0 .\end{cases}
$$


In particular, the set of integers $\left(a_{0}, a_{1}, \ldots, a_{\nu}\right)$ satisfy

$$
\left(\mathrm{SI}_{\text {red }}\right) \quad \begin{cases}a_{0} \geq 0 ;, \alpha_{l}>a_{l} \geq 0 & (1 \leq l \leq \nu) ; \\ 1+a_{0}+i e_{0}+\sum_{l}\left[\frac{i \omega_{l}+a_{l}}{\alpha_{l}}\right] \leq 0 & \text { for any } i>0 .\end{cases}
$$

Moreover, there is a one-to-one correspondence between the integer solutions of (SI), respectively of $\left(\mathrm{SI}_{r e d}\right)$, provided by the correspondence (2) above (see also Section [10.3).

Proof The first two set of inequalities follow from (SI) in Lemma 10.3.1 For the last one, we proceed as follows. For any $i>0$ we define $y(i):=i b_{0}+$ $\sum_{l, j} u_{j}^{l} b_{j}^{l} \in L$, where

$$
u_{1}^{l}:=\left[\frac{i n_{2 s_{l}}^{l}+\widetilde{a}_{1}^{l}}{n_{1 s_{l}}^{l}}\right], u_{2}^{l}:=\left[\frac{u_{1}^{l} n_{3 s_{l}}^{l}+\widetilde{a}_{2}^{l}}{n_{2 s_{l}}^{l}}\right], \cdots, u_{s_{l}}^{l}:=\left[\frac{u_{s_{l}-1}^{l}+\widetilde{a}_{s_{l}}^{l}}{n_{s_{l} s_{l}}^{l}}\right] .
$$

Consider $v:=l_{[k]}^{\prime}-y(i)$. The point is that $\left(v, b_{j}^{l}\right) \leq 0$ for any $1 \leq l \leq \nu$ and $1 \leq j \leq s_{l}$. Indeed, if $j<s_{l}$, from the definition of $u_{j}^{l}$ one has $u_{j}^{l} n_{j, s_{l}}^{l} \leq$ $u_{j-1}^{l} n_{j+1, s_{l}}^{l}+\widetilde{a}_{j}^{l}$, or, using Section 10.2 (namely $n_{j, s_{l}}^{l}=k_{j} n_{j+1, s_{l}}^{l}-n_{j+2, s_{l}}^{l}$ ) and $\widetilde{a}_{j}^{l}=\widetilde{a}_{j+1}^{l}+n_{j+1, s_{l}}^{l} a_{j}^{l}$,

$$
-a_{j}^{l}-u_{j-1}^{l}+k_{j} u_{j}^{l} \leq \frac{u_{j}^{l} n_{j+2, s_{l}}^{l}+\widetilde{a}_{j+1}^{l}}{n_{j+1, s_{l}}^{l}},
$$

hence $-a_{j}^{l}-u_{j-1}^{l}+k_{j} u_{j}^{l} \leq$ the integral part of the right hand side, which equals $u_{j+1}^{l}$. In other words, $\left(v, b_{j}^{l}\right)=-a_{j}^{l}-u_{j-1}^{l}+k_{j} u_{j}^{l}-u_{j+1}^{l} \leq 0$. (The case $j=s_{l}$ is easy.)

Since $v=l_{[k]}^{\prime}-y(i) \in l_{[k]}^{\prime}+L$ and $y(i)>0$, by the minimality of $l_{[k]}^{\prime}$ in $\left(l_{[k]}^{\prime}+L\right) \cap S_{\mathbb{Q}}$ we deduce that $v \notin S_{\mathbb{Q}}$. Since $\left(v, b_{j}^{l}\right) \leq 0$ for any $l$ and $j$, we get that $\left(v, b_{0}\right)>0$. This is equivalent to $-a_{0}-i e_{0}-\sum_{l} u_{1}^{l}>0$, hence the third inequality of $(S I)$ follows.

For $\left(\mathrm{SI}_{\mathrm{red}}\right)$, notice that $a_{l}=\widetilde{a}_{1}^{l}<n_{1 s_{l}}^{l}=\alpha_{l}$ from (SI). The correspondence between the integral solutions of the two system of inequalities is clarified in Section 10.3. namely, is given by $E\left(a_{l}\right)=\left(a_{1}^{l}, \ldots, a_{s_{l}}^{l}\right)$.

The point is that the set of solutions of (SI) (or, equivalently, of $\left(\mathrm{SI}_{\text {red }}\right)$ ) corresponds exactly to the set of all possible $\operatorname{spin}^{c}$-structures. In other words, if $\left\{a_{0} ; a_{j}^{l}\right\}_{j l}$ satisfies $\left(\mathrm{SI}_{\mathrm{red}}\right)$, then it is the set of coefficients of some $l_{[k]}^{\prime}$. This follows from the next fact. 
11.6 Proposition For any $c_{0} \geq 0$ and $\alpha_{l}>c_{l} \geq 0$ consider $E\left(c_{l}\right)=$ $\left(c_{1}^{l}, \ldots, c_{s_{l}}^{l}\right)$ as in Section [10.3. This defines $l^{\prime}:=-c_{0} g_{0}-\sum_{l, j} c_{j}^{l} g_{j}^{l} \in S_{\mathbb{Q}}$. If $l^{\prime}$ is not minimal in $\left(l^{\prime}+L\right) \cap S_{\mathbb{Q}}$ then for some $i>0$ one has

$$
1+c_{0}+i e_{0}+\sum_{l}\left[\frac{i \omega_{l}+c_{l}}{\alpha_{l}}\right]>0 .
$$

Proof Define the integers $\widetilde{c}_{j}^{l}, u_{j}^{l}$ and the cycle $y(i)$ (for any $i>0$ ) in a similar way as in (SI) and in previous proof, by replacing $a_{j}^{l}$ by $c_{j}^{l}$. Then the argument of the previous proof repeated gives that $y(i)$ satisfies

(a) $m_{0}(y(i))=i$, and

(b) $\left(l^{\prime}-y(i), b_{j}^{l}\right) \leq 0$ for any $l$ and $j$.

The point is that for any fixed $i>0, y(i)$ is the unique maximal effective cycle which satisfy both (a) and (b). This follows from the very definition of the integers $u_{j}^{l}$, its proof is similar to the proof of Proposition 11.11 and it is left to the reader.

Now, assume that $l^{\prime}=l_{[k]}^{\prime}+x$ for some effective non-zero cycle $x \in L$. Set $i:=m_{0}(x)$. Since $x$ satisfy both (a) and (b) for this $i$, from the maximality of $y(i)$ one gets $y(i) \geq x$. Consider now $l^{\prime}-y(i)=l_{[k]}^{\prime}+x-y(i)$. Since $\left(l^{\prime}-y(i), b_{j}^{l}\right) \leq 0$ one gets that $\left(l_{[k]}^{\prime}+x-y(i), b_{j}^{l}\right) \leq 0$ as well. Moreover, $\left(l_{[k]}^{\prime}+x-y(i), b_{0}\right) \leq\left(l_{[k]}^{\prime}, b_{0}\right) \leq 0$ (the first inequality from $b_{0} \notin|y(i)-x|$ and $x-y(i) \leq 0$, the second from $\left.l_{[k]}^{\prime} \in S_{\mathbb{Q}}\right)$. Hence $l_{[k]}^{\prime}+x-y(i) \in S_{\mathbb{Q}}$ with $x-y(i) \leq 0$. From the minimality of $l_{[k]}^{\prime}$ one gets $x=y(i)$. Hence $\left(b_{0}, l^{\prime}-y(i)\right)=\left(b_{0}, l_{[k]}^{\prime}\right) \leq 0$, or $-c_{0}-i e_{0}-\sum_{l}\left[i \omega_{l}+c_{l} / \alpha_{l}\right] \leq 0$.

11.7 Corollary The following sets are in one-to-one correspondence:

(1) $\operatorname{Spin}^{c}(M)$, or equivalently, the set of distinguished representatives $k_{r}=$ $K+2 l_{[k]}^{\prime}$;

(2) the set of integral solutions $\left\{a_{0} ; a_{j}^{l}\right\}_{l j}$ of (SI);

(3) the set of integral solutions $\left\{a_{0} ; a_{1}, \ldots, a_{\nu}\right\}$ of ( $\left.\mathrm{SI}_{\text {red }}\right)$.

The set $\left\{a_{0} ; a_{j}^{l}\right\}_{l j}$ is the coefficient set of $l_{[k]}^{\prime},\left\{a_{0}, \ldots, a_{\nu}\right\}$ is obtained from $\left\{a_{0} ; a_{j}^{l}\right\}_{l j}$ by Equation (2) of Section [11.4, Notice also that in $L^{\prime}$ one has

$$
-l_{[k]}^{\prime} \equiv a_{0} g_{0}+\sum_{l, j} a_{j}^{l} g_{j}^{l} \equiv a_{0} g_{0}+\sum_{l} a_{l} g_{s_{l}}^{l} \quad(\operatorname{modulo} L) .
$$

In particular, the cardinality of all these sets is the same $|H|=|e| \alpha_{1} \cdots \alpha_{\nu}$. The author knows no direct proof of this fact for (2) or (3). 
11.8 The values $\chi\left(l_{[k]}^{\prime}\right)$ Assume that $[k]$ corresponds (by the correspondence in Corollary 11.7) to the set $\left\{a_{0} ; a_{1}, \ldots, a_{\nu}\right\}$. Assume that $a_{l}>0$ for some fixed $1 \leq l \leq \nu$. Let $\left[k_{l}^{-}\right]$be the $\operatorname{spin}^{c}$-structure corresponding to

$$
\left\{a_{0} ; a_{1}, \ldots, a_{l-1}, a_{l}-1, a_{l+1}, \ldots, a_{\nu}\right\}
$$

(clearly, this set of integers also satisfies $\left.\left(\mathrm{SI}_{\text {red }}\right)\right)$. Then, by a similar method as in (the proof of) Proposition 10.5.1 we get

$$
\chi\left(l_{\left[k_{l}^{-}\right]}^{\prime}\right)=\chi\left(l_{[k]}^{\prime}\right)+\chi\left(g_{s_{l}}^{l}\right)+\frac{a_{0}}{e \alpha_{l}}+\sum_{t \neq l} \frac{a_{t}}{e \alpha_{t} \alpha_{l}}+\sum_{t \geq 1} a_{t}^{l}\left(g_{t}^{l}, g_{s_{l}}^{l}\right) .
$$

By a calculation (using Lemma 10.4 and Proposition 10.5.1):

$$
\sum_{t \geq 1} a_{t}^{l}\left(g_{t}^{l}, g_{s_{l}}^{l}\right)=\sum_{t \geq 1} a_{t}^{l}\left(\frac{n_{t+1, s_{l}}^{l}}{e \alpha_{l}^{2}}-\frac{n_{1, t-1}^{l}}{\alpha_{l}}\right)=\frac{a_{l}}{e \alpha_{l}^{2}}-\left\{\frac{a_{l} \omega_{l}^{\prime}}{\alpha_{l}}\right\} .
$$

Therefore,

$$
\chi\left(l_{\left[k_{l}^{-}\right]}^{\prime}\right)-\chi\left(l_{[k]}^{\prime}\right)=\chi\left(g_{s_{l}}^{l}\right)+\frac{1}{e \alpha_{l}}\left(a_{0}+\sum_{t=1}^{\nu} \frac{a_{t}}{\alpha_{t}}\right)-\left\{\frac{a_{l} \omega_{l}^{\prime}}{\alpha_{l}}\right\} .
$$

Hence, by induction (by decreasing the coefficients $\left(a_{1}, \ldots, a_{\nu}\right)$ to $\left.(0, \ldots, 0)\right)$, $\chi\left(l_{[k]}^{\prime}\right)$ can be computed in terms of $\chi\left(l_{\left[-a_{0} g_{0}\right]}^{\prime}\right)$. But, using the identities in Section 11.1, one has:

$$
-\chi\left(l_{\left[-a_{0} g_{0}\right]}^{\prime}\right)=\frac{1}{2}\left(-a_{0}\left(K, g_{0}\right)+a_{0}^{2} g_{0}^{2}\right)=\frac{a_{0}^{2}}{2 e}+\frac{a_{0}}{2}(1+\varepsilon) .
$$

Summing up these identities, and using $\widetilde{a}:=a_{0}+\sum_{l} a_{l} / \alpha_{l}$, one gets

$$
-\chi\left(l_{[k]}^{\prime}\right)=\sum_{l=0}^{\nu} \frac{a_{l}}{2}+\frac{\varepsilon \widetilde{a}}{2}+\frac{\widetilde{a}^{2}}{2 e}-\sum_{l=1}^{\nu} \sum_{i=1}^{a_{l}}\left\{\frac{i \omega_{l}^{\prime}}{\alpha_{l}}\right\} .
$$

For another expression see $(\mathrm{r} *)$ in Section 11.15.

11.9 The invariant $k_{r}^{2}+s$ Recall that $k_{r}^{2}+s=K^{2}+s-8 \chi\left(l_{[k]}^{\prime}\right) \cdot \chi\left(l_{[k]}^{\prime}\right)$ is computed above in Section 11.8, and $K^{2}+s$ in [12, Section 5.5]:

$$
K^{2}+s=\varepsilon^{2} e+e+5-12 \sum_{l=1}^{\nu} s\left(\omega_{l}, \alpha_{l}\right) .
$$

11.10 The cycles $x(i)$ for $i \geq 0$ We fix a $\operatorname{spin}^{c}$-structure $[k]$ which corresponds to the set $\left\{a_{0} ; a_{1}, \ldots, a_{\nu}\right\}$ by the correspondence in Corollary 11.7 Recall that the graded root $\left(R_{k_{r}}, \chi_{k_{r}}\right)$, in particular the $\mathbb{Z}[U]$-module $\mathbb{H}\left(R_{k_{r}}, \chi_{k_{r}}\right)$, can be completely recovered from the cycles $x(i)(i \geq 0)$ (see Sections 7 and 9). The next result determines these cycles in terms of the Seifert invariants of $M$ and the integers $\left\{a_{0}, \ldots, a_{\nu}\right\}$. 
11.11 Proposition For any $l$ consider $E\left(a_{l}\right)=\left(a_{1}^{l}, \ldots, a_{s_{l}}^{l}\right.$ ) (see Proposition 11.5 and Section 10.3), and define $\widetilde{a}_{j}^{l}:=\sum_{t \geq j} n_{t+1, s_{l}}^{l} a_{t}^{l}$ (for $1 \leq j \leq s_{l}$ ) (see (SI) in Proposition 11.5).

(1) Define the integers $\left\{v_{j}^{l}\right\}\left(1 \leq l \leq \nu, 1 \leq j \leq s_{l}\right)$ inductively by

$$
v_{1}^{l}:=\left\lceil\frac{i \omega_{l}-a_{l}}{\alpha_{l}}\right\rceil=\left\lceil\frac{i n_{2, s_{l}}^{l}-\widetilde{a}_{1}^{l}}{n_{1, s_{l}}^{l}}\right\rceil ; \quad v_{j}^{l}:=\left\lceil\frac{v_{j-1}^{l} n_{j+1, s_{l}}^{l}-\widetilde{a}_{j}^{l}}{n_{j, s_{l}}^{l}}\right\rceil\left(1<j \leq s_{l}\right) .
$$

Set $z(i):=i b_{0}+\sum_{l, j} v_{j}^{l} b_{j}^{l}$. Then $\left(l_{[k]}^{\prime}+z(i), b_{j}^{l}\right) \leq 0$ for any $l$ and $j$.

(2) Assume that an effective cycle $z(i):=i b_{0}+\sum_{l, j} v_{j}^{l} b_{j}^{l}$ (where $\left\{v_{j}^{l}\right\}_{l j}$ are some integers) satisfies $\left(l_{[k]}^{\prime}+z(i), b_{j}^{l}\right) \leq 0$ for any $l$ and $j$. Then

$$
v_{1}^{l} \geq\left\lceil\frac{i \omega_{l}-a_{l}}{\alpha_{l}}\right\rceil \text { and } v_{j}^{l} \geq\left\lceil\frac{v_{j-1}^{l} n_{j+1, s_{l}}^{l}-\widetilde{a}_{j}^{l}}{n_{j, s_{l}}^{l}}\right\rceil\left(1<j \leq s_{l}\right) .
$$

(3) In particular, $z(i)$ defined in (1) equals $x(i)$, the minimal effective cycle which satisfies $\left(l_{[k]}^{\prime}+x(i), b_{j}^{l}\right) \leq 0$ for any $l$ and $j$.

Proof The proof of (1) has the same spirit as the proof of Proposition 11.5. From the definition of $v_{j}^{l}$ one gets $n_{j, s_{l}}^{l} v_{j}^{l} \geq v_{j-1}^{l} n_{j+1, s_{l}}^{l}-\widetilde{a}_{j}^{l}$, which is equivalent to

$$
k_{j}^{l} v_{j}^{l}-v_{j-1}^{l}+a_{j}^{l} \geq \frac{v_{j}^{l} n_{j+2, s_{l}}^{l}-\widetilde{a}_{j+1}^{l}}{n_{j+1, s_{l}}^{l}} .
$$

Now, using the definition of $v_{j+1}^{l}$ for the right hand side, one gets $-a_{j}^{l}+$ $v_{j-1}^{l}-k_{j}^{l} v_{j}^{l}+v_{j+1}^{l} \leq 0$. (Here, if $j=1$ then $v_{j-1}^{l}:=i$.) For (2), notice that $\left(l_{[k]}^{\prime}+x(i), b_{s_{l}}^{l}\right) \leq 0$ is equivalent with the wanted inequality for $j=s_{l}$. In general, for arbitrary $j$, the inequality follows from $\left(l_{[k]}^{\prime}+x(i), v_{j}\right) \leq 0$, where $v_{j}=\sum_{t \geq j} n_{t+1, s_{l}}^{l} b_{t}^{l}$.

11.12 The values $\tau(i)(i \geq 0)$ Recall that $\tau(i):=\chi_{k_{r}}(x(i))(i \geq 0)$. If $i=0$ then $x(0)=0$, hence $\tau(0)=0$. From Lemma 9.1 part (c) and Proposition 11.11, one gets that for $i \geq 0$

$$
\tau(i+1)-\tau(i)=1-\left(l_{[k]}^{\prime}+x(i), b_{0}\right)=1+a_{0}-i e_{0}+\sum_{l}\left[\frac{-i \omega_{l}+a_{l}}{\alpha_{l}}\right] .
$$

In particular, for $i=0$ one has $\tau(1)-\tau(0)=1+a_{0} \geq 1$. In general,

$$
\tau(i)=\sum_{t=0}^{i-1}\left(1+a_{0}-t e_{0}+\sum_{l}\left[\frac{-t \omega_{l}+a_{l}}{\alpha_{l}}\right]\right) .
$$


11.13 The Ozsváth-Szabó invariant Using the general results Proposition 4.7. Theorem 8.3 and Theorem 9.3 , and

$$
\min \chi_{k_{r}}=\min _{i \geq 0} \tau(i)
$$

one gets

$$
H F_{\text {even }}^{+}(-M,[k])=\mathbb{H}^{+}(\Gamma,[k])=\mathbb{H}\left(R_{\tau}, \chi_{\tau}\right)\left[-\frac{k_{r}^{2}+s}{4}\right] .
$$

Clearly, from its very definition, the graded root $\left(R_{\tau}, \chi_{\tau}\right)$ is completely determined from the values $\{\tau(i)\}_{i \geq 0}$. Moreover, the numerical invariants can be determined as follows:

$$
\pm d( \pm M,[k])=\frac{k_{r}^{2}+s}{4}-2 \min _{i \geq 0} \tau(i),
$$

and (using Corollary [3.7 as well):

$$
\chi\left(H F^{+}(-M,[k])\right)=\min _{i \geq 0} \tau(i)+\sum_{i \geq 0} \max \left\{0,-1-a_{0}+i e_{0}-\sum_{l=1}^{\nu}\left[\frac{-i \omega_{l}+a_{l}}{\alpha_{l}}\right]\right\} .
$$

(Since $e=e_{0}+\sum \omega_{l} / \alpha_{l}<0$, this sum is finite.)

11.14 Remark Assume that $[k]=[K]$, ie $l_{[k]}^{\prime}=0$ (or, equivalently, $a_{0}=$ $\cdots=a_{\nu}=0$ ). Then the above sum becomes (the Dolgachev-Pinkham) invariant

$$
D P:=\sum_{i \geq 0} \max \left\{0,-1+i e_{0}-\sum_{l}\left[\frac{-i \omega_{l}}{\alpha_{l}}\right]\right\},
$$

and the above identity

$$
\chi\left(H F^{+}(-M, c a n)\right)-\min \chi_{\text {can }}=D P .
$$

This is interesting for two reasons.

(1) If $M$ is the link of a normal surface singularity with geometric genus $p_{g}$, then Corollary 9.6 reads as $p_{g} \leq D P$. In fact, if $M$ is a rational homology sphere, and it is the link of a singularity which admits a good $\mathbb{C}^{*}$-action (a fact which automatically implies that $M$ is a Seifert 3-manifold), then by results of Dolgachev and Pinkham, $p_{g}=D P$ (see eg [22]). It is remarkable, that in that context, the expression $D P$ is deduced by a rather different argument (sitting in algebraic geometry).

This inequality (and identity for singularities with good $\mathbb{C}^{*}$-action) is compatible with the conjecture [12], see also [13] for this special situation. 
(2) The identity $\mathbf{s w}_{M, \text { can }}^{O S z}=\mathbf{s w}_{M \text {,can }}^{T C W}$ in this case $[k]=[K]$ is equivalent to

$$
\mathcal{T}_{M, \text { can }}(1)-\frac{\lambda(M)}{|H|}=\frac{K^{2}+s}{8}+D P .
$$

This identity was verified in [13. In the sequel we verify the corresponding identity valid for an arbitrary $\operatorname{spin}^{c}$-structure.

11.15 The identity $\mathbf{s w}_{M,[k]}^{O S z}=\mathbf{s w}_{M,[k]}^{T C W}$ Using the above facts, this identity is equivalent to

$$
\mathcal{T}_{M,[k]}(1)-\frac{\lambda(M)}{|H|}=\chi\left(H F^{+}(-M,[k])\right)-\min \chi_{k_{r}}+\frac{k_{r}^{2}+s}{8} .
$$

We fix a $\operatorname{spin}^{c}$-structure $[k]$ which corresponds to $\left\{a_{0} ; a_{1}, \ldots, a_{\nu}\right\}$ as above (see Corollary [1.7]). For the completeness of the description, we also need (see [13]):

$$
\hat{P}_{1}(t):=\frac{\left(t^{\alpha}-1\right)^{\nu-2}}{\prod_{l}\left(t^{\alpha / \alpha_{l}}-1\right)} .
$$

Then by [13] (see also Sections 5.1] and 10.7), and with the notation $\widetilde{a}=a_{0}+$ $\sum_{l} a_{l} / \alpha_{l}$ one has

$$
\mathcal{T}_{M,[k]}(1)=\lim _{t \rightarrow 1}\left(\sum_{i \geq \widetilde{a} / e} \max \left\{0,1+a_{0}-i e_{0}+\sum_{l}\left[\frac{-i \omega_{l}+a_{l}}{\alpha_{l}}\right]\right\} t^{o i+\alpha \tilde{a}}-\frac{1}{|H|} \hat{P}_{1}(t)\right) .
$$

Using the inequalities (SI) (see Proposition 11.5) one deduce that the contribution corresponding to the indices $i<0$ is zero. Then using $\max \{0, x\}-$ $\max \{0,-x\}=x$, we get that

$$
\mathcal{T}_{M,[k]}(1)-\chi\left(H F^{+}(-M,[k])\right)+\min \chi_{k_{r}}
$$

equals the limit

$$
L:=\lim _{t \rightarrow 1}\left(P_{[k]}(t)-\frac{1}{|H|} \hat{P}_{1}(t)\right)
$$

where

$$
P_{[k]}(t):=\sum_{i \geq 0}\left(1+a_{0}-i e_{0}+\sum_{l}\left[\frac{-i \omega_{l}+a_{l}}{\alpha_{l}}\right]\right) t^{o i+\alpha \tilde{a}}
$$

In particular, the identity $(*)$ is equivalent to $\lambda(M) /|H|+\left(k_{r}^{2}+s\right) / 8=L$. This identity was verified in [13] for the canonical $\operatorname{spin}^{c}$-structure (see Section [5.1). Therefore, it is enough to check a relative identity (the difference between the two identities corresponding to an arbitrary $[k]$ and the canonical $[K])$. Since $k_{r}^{2}+s=K^{2}+s-8 \chi\left(l_{[k]}^{\prime}\right)$, this relative identity is

$$
-\chi\left(l_{[k]}^{\prime}\right)=\lim _{t \rightarrow 1}\left(P_{[k]}(t)-P_{[K]}(t)\right) .
$$


Notice that once this identity is proved, it also provides a new formula for $\chi\left(l_{[k]}^{\prime}\right)$. In order to verify $(\mathrm{r} *)$ we consider the Laurent expansions of $P_{[k]}(t)$ with respect to $t^{o}-1$. They have a pole of order two, and the above limit shows that the part with negative exponents is independent of $[k]$. For $[K]$ it was determined in [13, hence one has:

$$
P_{[k]}(t)=\frac{-e}{\left(t^{o}-1\right)^{2}}+\frac{-e-e \varepsilon / 2}{t^{o}-1}+\text { higher order terms. }
$$

In the sequel we write $P(t) \sim Q(t)$ if $\lim _{t \rightarrow 1}(P(t)-Q(t))=0$.

In the verification of $(\mathrm{r} *)$, we consider the same inductive steps as in Section 11.8. First, we verify $(\mathrm{r} *)$ for $l_{[k]}^{\prime}=-a_{0} g_{0}$. Then (using $o=-e \alpha$ as well) one gets

$$
\begin{gathered}
P_{[k]}(t)-P_{[K]}(t)=P_{[K]}(t)\left(t^{\alpha a_{0}}-1\right)+\sum_{i \geq 0} a_{0} t^{o i+\alpha a_{0}} \\
\sim-\left(t^{\alpha a_{0}}-1\right)\left(\frac{e}{\left(t^{o}-1\right)^{2}}+\frac{e+e \varepsilon / 2}{t^{o}-1}\right)+\frac{a_{0} t^{\alpha a_{0}}}{1-t^{o}} \sim \frac{a_{0}^{2}}{2 e}+\frac{a_{0}}{2}(1+\varepsilon) .
\end{gathered}
$$

This is compatible with $\chi\left(l_{\left[-a_{0} g_{0}\right]}^{\prime}\right)$ given in Section 11.8 Next, consider $[k]$ and $\left[k_{l}^{-}\right]$as in Section 11.8. Notice that

$$
\left[\frac{-i \omega_{l}+a_{l}}{\alpha_{l}}\right]-\left[\frac{-i \omega_{l}+a_{l}-1}{\alpha_{l}}\right]
$$

is 1 if $-i \omega_{l}+a_{l}$ is divisible by $\alpha_{l}$, otherwise is zero. The divisibility is satisfied if $i=\left\{\omega_{l}^{\prime} a_{l} / \alpha_{l}\right\} \alpha_{l}+j \alpha_{l}$ for some $j \geq 0$. Therefore,

$$
\begin{gathered}
P_{[k]}(t)-P_{\left[k_{l}^{-}\right]}(t)=P_{[k]}(t)\left(1-t^{-\alpha / \alpha_{l}}\right)+\sum_{i \omega_{l} \equiv a_{l}} t^{o i+\alpha \tilde{a}-\alpha / \alpha_{l}} \\
\sim \frac{t^{o \alpha_{l}\left\{\frac{\omega_{l}^{\prime} a_{l}}{\alpha_{l}}\right\}+\alpha \tilde{a}-\frac{\alpha}{\alpha_{l}}}}{1-t^{o \alpha_{l}}}+\left(t^{-\frac{\alpha}{\alpha_{l}}}-1\right)\left(\frac{e}{\left(t^{o}-1\right)^{2}}+\frac{e+e \varepsilon / 2}{t^{o}-1}\right) \\
\sim \frac{1}{2}+\frac{\varepsilon}{2 \alpha_{l}}-\frac{1}{2 e \alpha_{l}^{2}}+\frac{\widetilde{a}}{e \alpha_{l}}-\left\{\frac{\omega_{l}^{\prime} a_{l}}{\alpha_{l}}\right\} .
\end{gathered}
$$

This agrees with $\chi\left(l_{\left[k_{l}^{-}\right]}^{\prime}\right)-\chi\left(l_{[k]}^{\prime}\right)$ from Section [11.8, hence $(\mathrm{r} *)$ and $(*)$ follow by induction.

11.16 Corollary Again, since $\sum_{[k]} \mathcal{T}_{M,[k]}(1)=0$, one gets

$$
\lambda(M)=\sum_{[k] \in \operatorname{Spin}^{c}(M)} \chi\left(H F^{+}(M,[k])\right)-\frac{d(M,[k])}{2} .
$$




\section{References}

[1] M Artin, Some numerical criteria for contractibility of curves on algebraic surfaces, Amer. J. Math. 84 (1962) 485-496 MathReview

[2] M Artin, On isolated rational singularities of surfaces, Amer. J. Math. 88 (1966) 129-136 MathReview

[3] RE Gompf, I A Stipsicz, An Introduction to 4-Manifolds and Kirby Calculus, Graduate Studies in Mathematics 20, Amer. Math. Soc. (1999) MathReview

[4] H Grauert, Über Modifikationen und exceptionelle analytische Mengen, Math. Ann. 146 (1962) 331-368 MathReview

[5] H B Laufer, On rational singularities, Amer. J. Math. 94 (1972) 597-608 MathReview

[6] H B Laufer, On minimally elliptic singularities, Amer. J. Math. 99 (1977) 1257-1295 MathReview

[7] C Lescop, Global Surgery Formula for the Casson-Walker Invariant, Annals of Mathematics Studies 140, Princeton University Press (1996) MathReview

[8] I Luengo, A Melle-Hernández, A Némethi, Links and analytic invariants of superisolated singularities, J. Algebraic Geom. 2 (1993) 1-23 MathReview

[9] A Némethi, "Weakly" Elliptic Gorenstein Singularities of Surfaces, Invent. Math. 137 (1999) 145-167 MathReview

[10] A Némethi, Five lectures on normal surface singularities, from: "Low dimensional topology (Eger 1996/Budapest 1998)", Bolyai Soc. Math. Stud. 8 (1999) 269-351 MathReview

[11] A Némethi, Line bundles associated with normal surface singularities, arXiv:math.AG/0310084

[12] A Némethi, L I Nicolaescu, Seiberg-Witten invariants and surface singularities, Geom. Topol. 6 (2002) 269-328 MathReview

[13] A Némethi, L I Nicolaescu, Seiberg-Witten invariants and surface singularities II (singularities with good $\mathbb{C}^{*}$-action), J. London Math. Soc. 69 (2004) 593-607 MathReview

[14] W D Neumann, A calculus for plumbing applied to the topology of complex surface singularities and degenerating complex curves, Trans. Amer. Math. Soc. 268 (1981) 299-344 MathReview

[15] W Neumann, Abelian covers of quasihomogeneous surface singularities, from: "Singularities Part 2 (Arcata Calif. 1981)", Proc. Sympos. Pure Math. 40 (1983) 233-244 MathReview

[16] L I Nicolaescu, Seiberg-Witten invariants of rational homology 3-spheres, Commun. Contemp. Math. 6 (2004) 833-866 MathReview 
[17] P S Ozsváth, Z Szabó, Holomorphic discs and topological invariants for closed three-spheres, Ann. of Math. (2) 159 (2004) 1027-1158 MathReview

[18] P S Ozsváth, Z Szabó, Holomorphic discs and three-manifold invariants: properties and applications, Ann. of Math. (2) 159 (2004) 1159-1245 MathReview

[19] P S Ozsváth, Z Szabó, Holomorphic triangle invariants and the topology of symplectic four-manifolds, Duke Math. J. 121 (2004) 1-34 MathReview

[20] P S Ozsváth, Z Szabó, Absolutely graded Floer homologies and intersection forms for four-manifolds with boundaries, Adv. Math. 173 (2003) 179-261 MathReview

[21] P S Ozsváth, Z Szabó, On the Floer homology of plumbed three-manifolds, Geom. Topol. 7 (2003) 185-224 MathReview

[22] H Pinkham, Normal surface singularities with $\mathbb{C}^{*}$ action, Math. Ann. 117 (1977) 183-193 MathReview

[23] J Stevens, Elliptic surface singularities and smoothing of curves, Math. Ann. 267 (1984) 239-249 MathReview

[24] V G Turaev, Torsion invariants of $\operatorname{Spin}^{c}$-structures on 3-manifolds, Math. Res. Lett. 4 (1997) 679-695 MathReview 